\title{
A Numerical Investigation of the Effects of Classical Phase Space Structure on a Quantum System
}

\author{
G. Ball, K. Vant and N. Christensen \\ Department of Physics, University of Auckland, Private Bag 92019 Auckland, \\ New Zealand
}

\begin{abstract}
We present a detailed numerical study of a chaotic classical system and its quantum counterpart. The system is a special case of a kicked rotor and for certain parameter values possesses cantori dividing chaotic regions of the classical phase space. We investigate the diffusion of particles through a cantorus; classical diffusion is observed but quantum diffusion is only significant when the classical phase space area escaping through the cantorus per kicking period greatly exceeds Planck's constant. A quantum analysis confirms that the cantori act as barriers. We numerically estimate the classical phase space flux through the cantorus per kick and relate this quantity to the behaviour of the quantum system. We introduce decoherence via environmental interactions with the quantum system and observe the subsequent increase in the transport of quantum particles through the boundary.
\end{abstract}

\section{Introduction}

Quantum chaos is a relatively new field, comprising the study of the quantized versions of systems which are classically chaotic. The current consensus is that quantum systems do not show sensitive dependence on initial conditions in the same way as classical systems. This would appear to disqualify them from being described as chaotic. However, classical chaos is also apparent in other aspects of a system's evolution, the quantum analogies of which are of great interest. In particular, these include Kolmogorov-Arnold-Moser (KAM) tori and cantori, the study of which is the focus of this paper. Our group has previously published experimental studies [1, 2, 3] of a particular, classically chaotic system containing KAM tori and cantori, which is realized experimentally in the quantum regime, and simulated both classically and quantum mechanically. This paper concentrates on the detailed results of our computer simulations.

KAM tori and cantori in a classical phase space are predicted to influence the corresponding quantum system. An unbroken KAM boundary will prohibit classical diffusion through it, while tunneling across the barrier is possible in a quantum system. When interaction terms in the perturbing Hamiltonian are sufficiently large as to break up the boundary and create a cantorus or turnstile, classical particles will quickly diffuse through that cantorus but the quantum wavefunction will be inhibited [4, 5, 6]. A heuristic model proposes that with the presence of a perturbing Hamiltonian, quantum diffusion is constrained when the classical phase space area escaping through the cantorus each period is $\sim \hbar$ [6, 7]. Even though the barrier has been broken, the quantum wave function still appears to tunnel through the cantorus.

The link between the quantum domain and the familiar classical world remains a hotly debated topic. The quantum-classical correspondence (QCC)

\footnotetext{
${ }^{1}$ Electronic address: n.christensen $@$ auckland.ac.nz
} 
principle requires that quantum mechanics contains the classical macroscopic limit. A promising approach to the question of quantum-classical correspondence is the study of decoherence - the analysis of the effect of coupling to the environment, which inevitably occurs in a real system, in terms of quantum coherence [8]. We introduce increased environmental interactions into our quantum simulations in order to test the hypothesis that the resulting behaviour will more closely resemble the classical system.

The structure of this paper is as follows. In Section 2 we introduce the specifically designed double-kicked rotor system, and present the results of a classical analysis. In Section 3 we show that this classical evolution can be well described by a simple random model. In Section the study the corresponding quantum behaviour and analyze the properties of the system using the Floquet method. In Section 同 we introduce decoherence into the quantum system by two different methods, and also generate Wigner functions in order to help understand the origin of classical behavior from a quantum system. Finally a summary is contained in Section 6. All the parameters used in our model were chosen so as to correspond with those used in our experiments [1, 2, 3].

\section{Classical Double-Kicked Rotor}

The original and most commonly studied system in quantum chaos is the $\delta$ kicked rotor. The observation of dynamical localization in the atomic optics realization of the $\delta$-kicked rotor [9, 10, 11] provided an important experimental link to the most studied system in Hamiltonian chaos. However, a periodic pulsed potential of finite time duration (as used in the $\delta$-kicked rotor experiments) produces a KAM boundary, which becomes more noticeable for longer pulse widths. If one wishes to study diffusion through a cantorus, a train of single pulses is not the best system. The classical phase space outside the first long-lived cantorus is not strongly chaotic and contains many regular regions which will inhibit particle diffusion. Hence we have studied the dynamics due to a train of double pulses. This system has been the subject of experimental investigation by our group in its atomic optics manifestation [1, 2, 3]. Figure 1 ] displays our double pulse train. We can write the dimensionless form of the Hamiltonian as

$$
H=\frac{p^{2}}{2}-K \cos \phi \sum_{n=-\infty}^{\infty} f(t-n)
$$

where $p$ is the dimensionless momentum conjugate to the coordinate $\phi$ and $f(t)$ specifies the temporal shape of the pulses. $K$ is the dimensionless 'kicking strength' which is the single parameter varied in our investigation of the classical system. The double pulse train Hamiltonian can be written as

$$
H=\frac{p^{2}}{2}-K \sum_{m=-\infty}^{\infty} a_{m} \cos (\phi-2 \pi m \tau)
$$

where $a_{m}=\frac{1}{10} \operatorname{sinc}\left(\frac{m \pi}{20}\right) \cos \left(\frac{m \pi}{10}\right)$ (with the sinc function defined as $\operatorname{sinc}(x)=$ $\sin (x) / x$ ). Each pulse is of width $\alpha / 2$ and the leading edge separation of the two pulses is given by $\Delta$. The KAM boundaries at $p= \pm 10 \pi$ and $\pm 30 \pi$ correspond to zero values for $a_{5}$ and $a_{15}$. 
As discussed in [1], below a critical kicking strength $K=K^{*}$, the phase space of the classical system contains KAM tori given approximately by the lines $p= \pm 10 \pi$ for our choice of $\alpha=\Delta=0.1$. For $K>K^{*}$ these break up to become partially penetrable cantori. Figure 2 shows the phase space for $K=70$. We observe chaotic seas on either side of the cantorus around $p=10 \pi$. Figure 3 shows the phase space for $K=280$. We have stronger chaos with little island structure remaining. The KAM torus around $p=30 \pi$ is still unbroken for this kick strength, and we find that this is the case for all kicking strengths studied in this paper. The strongly chaotic seas surrounding the $p=10 \pi$ cantorus provide an ideal phase space structure for studying the transport of particles through the barrier.

For a system kicked by finite length pulses, numerical solutions for the motion are considerably more difficult to generate than for $\delta$-kicks. If the pulses are rectangular, then the system alternates between the motion of a pendulum, and free rotation. The solutions for pendulum motion are in terms of Jacobi elliptic functions and elliptic integrals. In our simulations, the elliptic integrals are efficiently evaluated with a specialized algorithm coded in C [12], which is then interfaced with MATLAB.

The starting point for the calculation of the momentum distributions is an initial distribution uniform in $\phi$, and Gaussian in $p$. For our chosen $\alpha$ and $\Delta$ we have $\sigma_{p}=3.6 \pi$ which gives $99.5 \%$ of initial conditions inside $p=|10 \pi|$. We find that the results are not strongly dependent on initial distribution, provided this proportion is close to 1 . To obtain numerical distributions, we choose $10^{5}$ initial conditions randomly from this distribution, and propagate them through 70 kick cycles. Figure 4 shows examples of our simulated distributions. Once the KAM boundaries at $p= \pm 10 \pi$ are broken the classical particles will eventually distribute themselves uniformly between the $p= \pm 30 \pi$ tori.

\section{Diffusion Model}

A very simple model, which mimics some aspects of the classical momentum distributions, is obtained by treating the system as consisting of three regions where strong, homogeneous diffusion occurs, divided by two permeable barriers. The system is enclosed by unbroken barriers. Applying this model to our system, we then assume that after a short time, the distributions both inside and outside the permeable barriers are each essentially uniform. We also assume that over time, the populations can leak across the penetrable barriers. The system can then be described by three variables giving the population associated with each region. If we choose our initial condition so that the population in each of the two 'outer' regions is small, we expect the populations to decay exponentially towards equilibrium values where the overall distribution is completely uniform.

We now take the barriers to be located at $p= \pm 10 \pi$. The outer boundaries are at $\pm 30 \pi$, so the system is divided into three equal parts. Each has a phase space area (in dimensionless units) of $40 \pi^{2}$. With each kick, some area of phase space $F$ is mapped from inside the cantori to above $p=10 \pi$. This area is referred to as the 'phase space flux per kick' through the $p=10 \pi$ cantorus. Identical amounts of phase space are mapped from above $p=10 \pi$ to inside, from inside to below $p=-10 \pi$, and from below $p=-10 \pi$ back inside. This follows from the incompressibility of phase space flow in a Hamiltonian system 
and the symmetry of this system in $p$. The assumption of separate uniform distributions inside each of the three intervals means that the probability of a trajectory inside the cantori being mapped outside is $2 F / 40 \pi^{2}$. The probability for a trajectory outside the cantori to be mapped inside is $F / 40 \pi^{2}$. Now let $P(|p|<10 \pi, t)$ be the probability for a particular trajectory to be inside the cantori. Take the initial condition to be $P(|p|<10 \pi, 0)=1$, so that both outside regions are empty. The evolution will maintain the symmetry between them so that the probability that the trajectory is in a particular outside region is $P(p>10 \pi, t)=P(p<10 \pi, t)=(1-P(|p|<10 \pi, t)) / 2$. The change in the distribution caused by one kick is

$$
\begin{aligned}
P(|p|<10 \pi, t+1)-P(|p|<10 \pi, t) & =-\frac{2 F}{40 \pi^{2}} P(|p|<10 \pi, t)+\frac{F}{40 \pi^{2}}(1-P(|p|<10 \pi,(t)) \\
& =-\frac{3 F}{40 \pi^{2}}\left(P(|p|<10 \pi, t)-\frac{1}{3}\right) .
\end{aligned}
$$

We can write this as

$$
P(|p|<10 \pi, t+1)-\frac{1}{3}=\left(1-\frac{3 F}{40 \pi^{2}}\right)\left(P(|p|<10 \pi, t)-\frac{1}{3}\right) .
$$

By induction, using our initial condition $P(|p|<10 \pi, 0)=1$, we have

$$
\begin{aligned}
P(|p|<10 \pi, t) & =\frac{1}{3}+\frac{2}{3}\left(1-\frac{3 F}{40 \pi^{2}}\right)^{t} \\
& =\frac{1}{3}+\frac{2}{3} e^{a t}
\end{aligned}
$$

where $a=\ln \left(1-3 F / 40 \pi^{2}\right) \approx-3 F / 40 \pi^{2}$ (a must be small for the model to apply). The probability for a trajectory to be outside the cantori is given by

$$
P(|p|>10 \pi, t)=\frac{2}{3}\left(1-e^{a t}\right) .
$$

The parameter $a$ is a function of kick strength which we do not know, except that it must increase with $K$. We can reverse the problem, estimating the flux $F$ by fitting an exponential decay to the proportion of trajectories outside $p= \pm 10 \pi$ versus $t$ for a particular kick strength. This estimate is informative for comparing classical and quantum results. Figure f(a) shows $P(|p|>10 \pi, t)$ versus $t$, for a range of kicking strengths while Figure 5 (b) shows $\ln [2 / 3-P(|p|>$ $10 \pi, t)$ ], which should be a straight line according to the simple diffusion model (after a few kicks).

We see that the model appears to be reasonably good for $K \leq 280$. For higher values of $K$ it may be that the transport across the barrier is too fast for equilibrium to approximately hold in each region. Also, in the real system, the equilibrium population outside will differ slightly from $2 / 3$, so that the straight line form of our graph will break down for $P(|p|<10 \pi)$ very close to equilibrium. Inspection suggests that the real equilibrium value differs from $2 / 3$ by $\lesssim e^{-3} \approx 0.05$. Figure 6 shows estimates of the flux per kick $F$ through the cantori based on fitting straight lines to the curves in Figure 5 . 


\section{Quantum Momentum Distributions and Flo- quet States}

For comparison with classical simulations, we use an initial density matrix

$$
\left\langle m\left|\widehat{\rho}_{0}\right| n\right\rangle=\frac{1}{A} \exp \left(-\frac{n^{2} \hbar^{2}}{2 \sigma_{p}^{2}}\right) \delta_{m, n}
$$

where $\sigma_{p}=3.6 \pi, A$ is a normalization constant and $k$ is Planck's constant in our dimensionless system. Again the results are not strongly dependent on the

initial spread. We simulate the system dynamics for up to 70 kicks. Figure 7 shows examples of these distributions, for the same kicking strengths as were presented in the classical case, with $k=2.6$. Figure 8 (a) shows the quantum probability for the atom to be outside $p=|10 \pi|$ versus $t$, for a range of kicking strengths. Figure $\mathbb{8}$ (b) shows $\ln [2 / 3-P(|p|>10 \pi, t)]$.

Comparing these graphs to Figure 5 we see that, for all of these kicking strengths, the quantum behaviour deviates qualitatively from the classical after less than 20 kicks, and the probability outside the boundary for a quantum system always levels off well below the classical equilibrium value. This is consistent with the premise that the diffusion is suppressed when the phase space flux across the cantorus is $\lesssim \hbar$. This KAM localization is distinct from the more widely studied dynamical localization [1].

The time evolution operator $U$ is unitary, so that its eigenvalues are of the form $\alpha_{j}=\exp \left(-i E_{j} / \hbar\right)$ where $E_{j}$ is a (dimensionless) quasi-energy. Its eigenstates (quasi-energy states or Floquet states) satisfy

$$
U\left|\alpha_{j}\right\rangle=e^{-i E_{j} / \hbar}\left|\alpha_{j}\right\rangle .
$$

In a basis made up of these states, the evolution operator is diagonal. They are therefore equivalent to eigenstates of the Hamiltonian in a time-independent system, and if we examine the system only once per kicking cycle, they are effectively stationary. If we are interested in the state of the system after quantum saturation has occurred, we can examine the asymptotic (long-time average) momentum distribution, which can be written

$$
\begin{aligned}
P\left(n \mid \rho_{0}\right) & =\lim _{N \rightarrow \infty} \frac{1}{N} \sum_{t=0}^{N-1}\left\langle n\left|\rho_{t}\right| n\right\rangle \\
& =\lim _{N \rightarrow \infty} \frac{1}{N} \sum_{t=0}^{N-1}\left\langle n\left|U^{t} \rho_{0} U^{\dagger t}\right| n\right\rangle .
\end{aligned}
$$

By inserting the spectral decomposition of $U$ (representation in terms of the Floquet states) we obtain [4, 政

$$
P\left(n \mid \rho_{0}\right)=\sum_{j}\left\langle\alpha_{j}\left|\widehat{\rho}_{0}\right| \alpha_{J}\right\rangle \cdot\left|\left\langle n \mid \alpha_{j}\right\rangle\right|^{2}
$$

and if our initial condition is a pure state $\widehat{\rho}_{0}=\left|n_{0}\right\rangle\left\langle n_{0}\right|$ we have

$$
P\left(n \mid n_{0}\right)=\sum_{j}\left|\left\langle n_{0} \mid \alpha_{j}\right\rangle\right|^{2} \cdot\left|\left\langle n \mid \alpha_{j}\right\rangle\right|^{2} .
$$


This function characterizes the asymptotic mixing between the two momentum states. We can generate pseudo-colour plots of $P\left(n \mid n_{0}\right)$, to obtain visual representations of the momentum confinement in the quantum system for particular choices of $K$ and $k$.

Figure 9 shows a series of these pseudo-colour plots. We have used $k=2.6$ and $N=128$, so that $k\left|n_{\max }\right|>50 \pi$. The plots use a logarithmic colour-scale. Black and dark grey regions correspond to vanishingly small probability. Mid grey regions indicate a low probability, at the level of quantum tunneling. Pale grey and white regions have a sizeable probability density. Inspection of the plots shows that their shape appears to be chiefly determined by the classical barriers to momentum diffusion. The light line along the line $p=p_{0}$ arises from each state being mapped onto itself, to some degree. Each plot also shows a central light square with $|p|$ and $\left|p_{0}\right|$ less than $10 \pi$, indicating the strong coupling of each state in this range to all the others. Sharp borders on this square indicate strong confinement by the cantori. These borders blur as the cantori become less effective. For increased kicking strength we eventually see a corresponding square associated with the KAM tori at $p= \pm 30 \pi$. Figure 9(a) has $K=80$ and we see very strong signatures of the classical barriers. Figure 9(b) and (c) show $K=180$ and $K=280$ respectively. We see significant penetration of the $p= \pm 10 \pi$ cantori, but their effect is still obvious. The $p= \pm 30 \pi$ KAM tori still provide a very strong barrier. Figure $9(\mathrm{~d})$ has $K=400$. The effects of the $p= \pm 10 \pi$ cantori have almost disappeared, but penetration through the $p= \pm 30 \pi$ KAM tori is still fairly small.

Again, we see strong localization which begins to break down for $K \approx 300$. Referring to Figure 6, we see that the classical flux through each cantorus becomes comparable with $k=2.6$ when $K \approx 250$. It has previously been observed [13] that this criterion appears to give a reasonable estimate of the kicking strength for which strong quantum localization by a cantorus will be destroyed.

\section{Decoherence via Spontaneous Emission}

In order to study decoherence we consider the atomic optics manifestation of this double kicked system [1, 2, 3]. A periodic potential is created by two counter-propagating laser beams, and atoms are subjected to a force due to the dipole potential. The dipole potential is derived by neglecting the excited state amplitude of the atom [14, 15]. We will now consider the first order effects arising from a small non-zero amplitude. Rather than making coherent stimulated transitions which is the usual interaction between the atoms and the pulsed potential, an atom in the excited state may, with finite probability, decay to the ground state by spontaneous emission. We can treat this effect as a stochastic process, which is different for each realization. The effect of a spontaneous emission event arises from the recoil momentum imparted to the atom by both the photon exciting the atom and the photon emitted by the atom. The atom recoils with a change in $p$ of $u k$ where $-1 \leq u \leq 1$ and $u=\frac{1}{2}( \pm 1+\cos \beta)$, where the upper and lower signs occur with equal probability and $\beta$ is the angle which the spontaneously emitted photon makes with the $x$ axis. $\beta$ is random, with a distribution which is a sum of dipole distributions over the set of possible atomic orientations. To a fairly good approximation [10], $u$ can be treated as uniformly distributed between -1 and 1 . We define 
$\eta$ to be the probability per kicking cycle that the atom undergoes spontaneous emission.

\subsection{Density Matrix Calculations}

To include spontaneous emission in our density matrix calculations, we use the following approximate technique. For a particular run we choose a probability per kicking cycle $\eta$ that a particular atom will spontaneously emit. Equivalently $\eta$ is the proportion of the atoms in the ensemble represented by the density matrix which will spontaneously emit in each cycle. We then discretise $u$, so that $u= \pm 1$ with equal probability. Therefore the recoil of an atom in state $|n\rangle$ will leave it in state $|n-1\rangle$ or $|n+1\rangle$. These states are representable by the density matrix, unlike those arising from continuous $u$. Once per kick, the following replacement is made:

$$
\langle m|\widehat{\rho}| n\rangle \leftarrow \frac{1}{2} \eta(\langle m+1|\widehat{\rho}| n+1\rangle+\langle m-1|\widehat{\rho}| n-1\rangle)+(1-\eta)\langle m|\widehat{\rho}| n\rangle
$$

where we apply periodic boundary conditions.

We have also performed Monte Carlo wavefunction simulations which are more realistic but considerably more time consuming. In particular they take into account the continuous distribution of recoil momenta in the $x$ direction, and the fact that spontaneous emission can occur at any time during the laser kick. The recoil due to spontaneous emission is continuous between $-k$ and $\hbar$, and alters the 'ladder' on which coherent dynamics occur for the particular atom. The approximate way in which we account for spontaneous emission in our density matrix calculation produces results which are negligibly different from the Monte Carlo wavefunction calculation, but are significantly more computationally efficient.

\subsection{Momentum Distributions}

Figures 10 and 11 show momentum distributions versus number of kicks for the quantum double-kicked rotor, with spontaneous emission effects included. Figure 10(a) shows behaviour for $K=80$ and $\eta=2 \%$. Comparing this to Figure (a), any broadening of the distributions due to the spontaneous emission is not obvious. However the 'spiky' nature of the pure quantum version has been significantly suppressed. Figure 11.a) has the same kicking strength and $\eta=5 \%$. Here we still see little broadening but even stronger suppression of the quantum peaks. Figure 10(b), with $K=180$ and $\eta=2 \%$, again shows similarity to the pure quantum case (in Figure 7(b)), except for some suppression of peaks and slight broadening. In Figure 11(b) has $K=180$ and $\eta=5 \%$. We now see unmistakable movement of probability into the wings of the distribution, qualitatively resembling that in the classical system for $K=180$, in Figure 4 (b), although not as strong. Figure 10 (c) has $K=280$ and $\eta=2 \%$. There is much more flow of probability through the cantori than in the pure quantum case in Figure 7(b), although the spikiness of the distribution is still strikingly

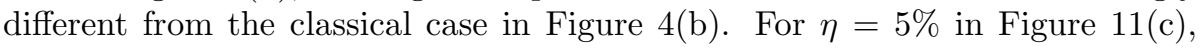
the distributions might be considered to look more classical than quantum, although the rate of transport through the boundary does not match the classical case. Finally for $K=400$, we again see (Figures 10(d) and 11(d)) a significant 
increase in the probability flow due to spontaneous emission, accompanied by distribution shapes which qualitatively resemble classical behaviour except for a somewhat smaller rate of transport.

\subsection{Wigner Functions}

A convenient way to visualize the information represented by the density matrix is in the form of a Wigner function. For a discrete, truncated basis we use the toroidal Wigner function as defined in [16],

$$
w\left(X_{k}, P_{l}, t\right)=\sum_{j=0}^{2 N-1} \exp \left(i \frac{\pi j k}{N}\right) \frac{1+(-1)^{l+j}}{2}\left\langle\frac{l+j}{2}|\widehat{\rho}| \frac{l-j}{2}\right\rangle
$$

where $P_{l}=(k / 2) l$ and $X_{k}=\pi k / N$. This gives a Wigner function defined on a $2 N \times 2 N$ grid. Averaging over cells of four adjacent points we reduce the grid to $N \times N$. This was implemented in MAtLAB using a fast Fourier transform algorithm.

Figures 12 through 19 show a series of three-dimensional graphs of Wigner functions. Each function represents the state of the quantum double-kicked system after 20 kicks, with the same initial condition as for our other simulations. Figure 12 has $K=80$ and no spontaneous emission. We see that the Wigner function is strongly contained by the classical barriers. It has a complicated folded shape with some sharp spikes. In Figure 13 we introduce spontaneous emission with rate $\eta=5 \%$. The Wigner function is qualitatively similar, but close comparison shows that it has become somewhat smoother, with the contrast between peaks and troughs being reduced. In Figure 14 we have $K=180$ and $\eta=0$. The Wigner function 'spills' over the classical barriers, although the total probability outside is small. We chiefly notice that the Wigner function is now much more complicated in shape, varying rapidly in position and momentum. There are significant negative peaks present. The addition of spontaneous emission in Figure 15 again serves to suppress this variation. It is not obvious from inspection of these graphs, but the cantori localization is also destroyed to some degree by finite $\eta$.

In Figure 16, with $K=280$ and $\eta=0$, we again see an increase in the complexity of the Wigner function. At this kicking strength, the effect of the KAM tori around $p= \pm 30 \pi$ has become apparent. The introduction of spontaneous emission in Figure 17 again serves to smooth this variation somewhat, while pushing more (quasi) probability into the wings of the function. Finally we examine $K=400$. For the situation without spontaneous emission (Figure 18) the function is again very complicated and noisy-looking in shape. The main visible change with increased kicking strength is the increase in the function near to the $p= \pm 30 \pi$ boundaries. Introduction of spontaneous emission (Figure 19) again suppresses the rapid variation to some extent.

In the limit of small $k$ we expect that the Wigner pseudo-probability distribution will tend to a classical probability distribution. The Wigner function itself cannot be interpreted as a probability distribution because it is not always positive. We can argue that the Wigner functions of states of particularly quantum character will exhibit this non-positivity strongly. The normalization 
of our discrete Wigner function is

$$
\sum_{k, l=1}^{N} \bar{W}\left(\phi_{k}, p_{l}\right)=1
$$

but due to non-positivity

$$
\sum_{k, l=1}^{N}\left|\bar{W}\left(\phi_{k}, p_{l}\right)\right| \geq 1
$$

We would like to quantify the 'non-classicality' of a given state with a single positive number. A possible choice is

$$
S=\sum_{k, l=1}^{N}\left(\left|\bar{W}\left(\phi_{k}, p_{l}\right)\right|-\bar{W}\left(\phi_{k}, p_{l}\right)\right) \geq 0 .
$$

We refer to $\mathrm{S}$ as 'quantum strangeness'. In this paper, we will not analyze this quantity in any detail. However, for example, a mixed state consisting of two Gaussian wave packets centered at $(\phi, p)=(0, \pm 16 k)$ has $S=0.1765$, while for a superposition state with equally weighted components of the two wavepackets $S=0.7647$. For the initial state we use for our simulations $S=0$. In general the larger the value of $S$, the more non-classical the character of the state. Figure 20 shows this parameter calculated for the state of our system after 20 kicks, for several kicking strengths $K$ and spontaneous emission rates $\eta=0,2 \%$ and $5 \%$.

\subsection{Decoherence Versus Heating}

An atom which undergoes spontaneous emission receives a random momentum kick. This will obviously lead to an increase in the width of the momentum distribution, the net effect being some incoherent absorption of energy from the laser beams. This effect is referred to as 'heating'. This however is not the main physical mechanism behind the increased diffusion we observe due to spontaneous emission. In Figure 21(a) and 21(b) we show the additional diffusion through $p= \pm 10 \pi$ over the pure quantum case, for several kicking strengths and two values of spontaneous emission rate $\eta$. We note that the contribution of heating to the crossing of this boundary will be strongest when the momentum distribution is steep near $p= \pm 10 \pi$. If the broadening effect was due entirely to heating it should be most pronounced for the smaller kicking strengths $K$ where the pure quantum distribution is strongly localized by the cantori, and for fixed $\eta$, should not increase with increasing $K$. Figure 21(a) shows that the additional diffusion caused by spontaneous emission rate $\eta=2 \%$ is much larger at $K=180,280$ and 400, than at $K=80$. Figure 21(b) shows the same trend for $\eta=5 \%$. There must therefore be another mechanism which increases the diffusion rate, and which is much stronger than the heating mechanism for $K \geq 180$. This mechanism is the destruction of quantum coherence, or decoherence, caused by the randomizing effect of spontaneous emission. We have also experimentally verified that heating is negligible for $K>150$ [1]. 


\subsection{Measurement Decoherence, or the Anti-Zeno Effect}

Our model for spontaneous emission leads to decoherence which we can consider to be 'environmentally induced'. Kaulakys and Contis [18] discuss the effect of projective momentum measurements on the dynamics of the quantum $\delta$-kicked rotor. They find that if a momentum measurement is made after every kick, then quantum localization is completely destroyed, and unbounded diffusion occurs, with the same diffusion constant as in the classical system. They refer to this modification of the dynamics as an anti-Zeno effect. Each measurement corresponds to the diagonalization of the density matrix in the momentum representation (i.e. off-diagonal elements become zero), or equivalently, the loss of all information about position. This effect is formulated without appealing to a collapse of the wave-function, and can in principle be produced experimentally.

We perform a similar simulation for our system to compare this form of decoherence to the spontaneous emission induced decoherence and to the classical motion. Computationally this is very similar to the density-matrix spontaneous emission simulations. After each double-kick coherent cycle, the density matrix is replaced by a matrix with the same diagonal entries, but all zero off-diagonal entries. The cycle then repeats. We note that the Wigner functions for states generated by this type of simulation are very simple, being the product of a uniform position distribution and the momentum distribution. We therefore do not show them, but point out that the 'quantum strangeness' parameter $S$ is always zero for these functions.

\subsection{Comparison of Quantum and Classical Results}

To compare our quantum and classical results, we calculate the probability for a particle to cross the $p= \pm 10 \pi$ cantori, $P(|p|>10 \pi, t)$, for classical, pure quantum, spontaneous emission and anti-Zeno simulations of the system. Figure 22(a) has $K=80$. Classical and pure quantum simulations give essentially horizontal lines with some fluctuation, because the KAM tori present at this kicking strength are effective barriers for both systems. The initial conditions determine the level of this line. The quantum simulations with spontaneous emission show a gradual leakage and the quantum anti-Zeno simulation gives a qualitatively similar result. This suggests that, at this kicking strength, decoherence breaks down the quantum cantorus localization, destroying the correspondence between the quantum and classical systems.

In Figure 22(b), we present simulations for $K=180$. There is now a clear difference between the classical and quantum simulations. In fact they appear to differ even for very small $t$, where the quantum curve initially rises more sharply than the classical, before saturation sets in. Adding spontaneous emission destroys the saturation, and for $t>10$ causes the quantum system to qualitatively more closely resemble the classical. The anti-Zeno simulation however suggests that the limit of large decoherence is again faster transport across the cantori than in the classical case. We note that this simulation does not involve heating effects, so the transport must be due to decoherence.

Now shifting our attention to $K=280$, in Figure 22(c), we see a large difference between classical and pure quantum behaviour which appears to be bridged by the introduction of spontaneous emission. The anti-Zeno calculation 
now closely corresponds to the classical picture, and we conclude that at this kicking strength decoherence does make the quantum system 'more classical'.

Finally we consider $K=400$ in Figure 22(d). The pure quantum case is not as far from the classical behaviour as for $K=280$, but again decoherence is effective in increasing the similarity between the systems. The anti-Zeno calculation now gives results that are almost indistinguishable from the classical. The close correspondence between the anti-Zeno and classical simulations for this and the previous figure can be related to the fact that for these kicking strengths the size of the classical flux per kick (in Figure 6) has become comparable to our dimensionless Planck's constant $k=2.6$.

Our group has previously published experimental results for $K=280$ [2]. These show good agreement with the spontaneous emission simulations, especially the Monte Carlo runs. With the elimination of some systematic experimental errors we expect that still better agreement would be achieved.

Figure 22 demonstrates that, as we have seen, when $\eta=0$, quantum saturation sets in by $t=20$ over our entire range of kicking strengths. If we examine the $\eta=0$ results we see that the quantum strangeness $S$ is very small for $K=80$, but rapidly increases with increasing kicking strength. In general, we expect to see $S$ increase with the chaoticity of the corresponding classical system. Classical systems with strong chaos rapidly develop phase space structure on all scales, which cannot be reproduced in a quantum system with finite $k$, so the quantum system must begin to exhibit non-classical features. It is interesting here that when $K=80, S$ is almost negligible, which seems to correspond to the fact that the classical and quantum systems are both strongly localized by the KAM tori in the classical system. As $K$ increases, the small scale phase space structure making up the cantori must differ in the classical and quantum systems, and we see that $S$ rises quickly with $K$ in this regime. Examining the effects of introducing spontaneous emission, we see that $S$ is significantly reduced even by a rate $\eta=2 \%$. Along with the 'more classical' diffusion seen in Figure 22, we can see that the Wigner function also exhibits more 'classicality' when we introduce environmental decoherence.

\section{Discussion}

We have analyzed and numerically simulated the classical double-kicked rotor system and verified that it possesses KAM tori and cantori which present barriers to diffusion. We have established the success of a simple diffusion model for the system and used it to estimate the phase space flux per kick through a cantorus as a function of kicking strength. The double-kicked rotor is an interesting chaotic system, which would reward further analysis. One aspect would be locating periodic trajectories of the system, especially the series of these trajectories converging to the noble KAM torus or cantorus.

The quantum double-kicked system shows strong localization corresponding to classical KAM tori. The system is also localized by classical cantori and does not show the sharp transition shown classically. Whereas the classical system eventually reaches a uniform distribution in phase space, the quantum system saturates with probability still significantly confined by the classical barriers. This saturation is confirmed by a Floquet analysis of asymptotic momentum distributions. The effect is still obvious even when the size of the classical 
flux per kick becomes of the order of our dimensionless Planck's constant, $k$. In addition to the saturation, we observe fluctuating peaks in the momentum distributions, which contrast strongly to the flat-topped distributions seen classically. The quantum transport through the classical boundary more closely resembles the classical situation as the kicking strength increases, but examination of the Wigner functions shows that the system becomes more non-classical. This is consistent with the general theory of quantum chaos which suggests that stronger chaos in the classical system accelerates the appearance of quantum coherence effects [10, 16, 19].

The modern theory of environmental decoherence states that the interaction of quantum systems with the environment is a necessary condition for the appearance of classical behaviour in real systems. The traditional semi-classical limit, which in our formulation is given by $k \rightarrow 0$, is unsatisfactory because the quantum break time becomes infinite only logarithmically, and can be surprisingly short for a real macroscopic system. After this time arbitrary quantum superpositions may arise.

We have observed the effects of decoherence on our quantum system, using two models; dissipation by spontaneous emission caused by the kicking laser beam, and the anti-Zeno effect. Where the classical flux per kick through the cantorus is finite, decoherence disrupts quantum saturation and the system is qualitatively more like the classical version. Examination of the Wigner function also indicates that quantum interference effects are suppressed by the decoherence. The anti-Zeno calculations are a kind of extreme decoherence limit, and we have seen that they reproduce the classical dynamics accurately, provided that $\hbar$ is not too large compared to the classical flux per kick. It appears not unreasonable to suppose that this correspondence continues indefinitely.

There are numerous aspects of decoherence in this system which could be further investigated. The introduction of noise into the kicking of the system is expected to have a similar effect to that of dissipation through spontaneous emission [20]. Particular states of the system will be 'resistant' to decoherence. These are expected to be the 'classical' states in the limit of strong decoherence and small $k$. We would like to determine some of these states and quantify the 'decoherence times' for other states, for comparison with the quantum break time of the coherent system. It would be interesting to quantitatively compare the anti-Zeno effect with decoherence via noise and dissipation.

Our results support the idea that the apparently classical nature of the universe arises entirely from quantum mechanics. In the real world the unpredictable behaviour of chaotic systems must ultimately arise from quantum uncertainty. As further progress is made in the investigation of decoherence, there is hope that physics will develop a consistent picture of a smooth transition between quantum and classical descriptions of reality.

\section{Acknowledgements}

This work was supported by the University of Auckland Research Committee and the Royal Society of New Zealand Marsden Fund.

\section{References}


[1] K. Vant, G. Ball, H. Ammann, and N. Christensen, Physical Review E 59, 2846 (1999).

[2] G. H. Ball, K. M. D. Vant, H. Ammann, and N. L. Christensen, In press, Journal of Quantum and Semiclassical Optics (1999), preprint quant$\mathrm{ph} / 9902006$.

[3] N. Christensen, H. Ammann, G. Ball, and K. Vant, Laser Physics 9, 281 (1999).

[4] T. Geisel, G. Radons, and J. Rubner, Physical Review Letters 57, 2883 (1986).

[5] T. Geisel and G. Radons, Physica Scripta 40, 340 (1989).

[6] R. C. Brown and R. E. Wyatt, Physical Review Letters 57, 1 (1986).

[7] R. S. MacKay and J. D. Meiss, Physical Review A 37, 4702 (1988).

[8] W. H. Zurek, Physics Today 44, 36 (1991).

[9] F. L. Moore et al., Physical Review Letters 75, 4598 (1995).

[10] H. Ammann, R. Gray, I. Shvarchuck, and N. Christensen, Physical Review Letters 80, 4111 (1998).

[11] H. Ammann, R. Gray, N. Christensen, and I. Shvarchuck, Journal of Physics B: Atomic, Molecular and Optical Physics 31, 2449 (1998).

[12] W. H. Press, S. A. Teukolsky, W. T. Vetterling, and B. P. Flannery, Numerical Recipes in $C$ : The Art of Scientific Computing, 2nd ed. (Cambridge University Press, New York, 1995).

[13] R. C. Brown and R. E. Wyatt, Physical Review Letters 57, 1 (1986).

[14] J. Dalibard and C. Cohen-Tannoudji, Journal of Optical Society of America B 2, 1707 (1985).

[15] R. Graham, M. Schlautmann, and P. Zoller, Physical Review A 45, R19 (1992).

[16] A. R. Kolovsky, Chaos 6, 534 (1996).

[17] K. M. D. Vant, MSc thesis (Physics), University of Auckland, Auckland, New Zealand, 1999.

[18] B. Kaulakys and V. Gontis, Physical Review A 56, 1131 (1997).

[19] W. H. Zurek and J. P. Paz, Physical Review Letters 72, 2508 (1994).

[20] B. G. Klappauf, W. H. Oskay, D. A. Steak, and M. G. Raizen, Physical Review Letters 81, 1203 (1998). 
Figure 1: Double pulse showing definitions of $\alpha$ and $\Delta$.

Figure 2: Poincare section for double-kicked rotor with $K=70$.

Figure 3: Poincare section for double-kicked rotor with $K=280$. The cantori at $p= \pm 10 \pi$ are no longer visible in the phase space at this resolution.

Figure 4: Classical momentum distribution as a function of number of kicks $t$, for the standard double-kicked system with (a) $K=80$, (b)180, (c)280 and (d) 400 .

Figure 5: (a) Proportion of trajectories outside $p= \pm 10 \pi$, versus number of kicks $t$. Kicking strength for each curve is indicated by the legend. (b) Logarithmic plot of $2 / 3-P(|p|>10 \pi)$, versus number of kicks $t$.

Figure 6: Estimate of phase space flux per kick $(\mathrm{F})$ through cantorus versus kicking strength $K$.

Figure 7: Quantum momentum distribution as a function of number of kicks $t$, with (a) $K=80$, (b)180, (c)280 and (d)400.

Figure 8: (a) Probability outside $p= \pm 10 \pi$, versus number of kicks $t$. Kicking strength $K$ for each curve is 120 (dotted), 150 (dashed), 180 (dot-dashed), 210 (solid), 250 (heavy dotted) and 280 (heavy dashed). (b) Logarithmic plot of $2 / 3-P(|p|>10 \pi)$, versus number of kicks $t$. Classically these curves are almost straight lines.

Figure 9: Pseudo-colour plot of asymptotic momentum distribution $P\left(p \mid p_{0}\right)$ for (a) $K=80$, (b)180, (c)280 and (d) 400, $k=2.6$. Computed using 128 states, upon which $P\left(p \mid p_{0}\right)$ is normalized to 1 .

Figure 10: Quantum momentum distribution as a function of number of kicks $t$ with $\eta=2 \%$ (a) $K=80$, (b)180, (c)280 and (d)400.

Figure 11: Quantum momentum distribution as a function of number of kicks $t$ with $\eta=5 \%$ (a) $K=80$, (b)180, (c)280 and (d)400.

Figure 12: Wigner function after 20 kicks with $K=80$ and no spontaneous emission.

Figure 13: Wigner function after 20 kicks with $K=80$ and $\eta=5 \%$.

Figure 14: Wigner function after 20 kicks with $K=180$ and $\eta=0$.

Figure 15: Wigner function after 20 kicks with $K=180$ and $\eta=5 \%$. 
Figure 16: Wigner function after 20 kicks with $K=280$ and $\eta=0$.

Figure 17: Wigner function after 20 kicks with $K=280$ and $\eta=5 \%$.

Figure 18: Wigner function after 20 kicks with $K=400$ and $\eta=0$.

Figure 19: Wigner function after 20 kicks with $K=400$ and $\eta=5 \%$.

Figure 20: 'Quantum strangeness' $S=\sum_{k, l=1}^{N}\left(\left|\bar{W}\left(\phi_{k}, p_{l}\right)\right|-\bar{W}\left(\phi_{k}, p_{l}\right)\right)$ versus kicking strength $K$ for spontaneous emission rates $\eta=0,2 \%, 5 \%$ as indicated in legend.

Figure 21: (a) Relative effect of spontaneous emission rate $\eta=2 \%$ for different kicking strengths $K=80$ (solid), 180 (dotted), 280 (dashed) and 400 (dotdashed). Proportion of atoms outside $p= \pm 10 \pi$ for quantum simulations with $\eta=0$ and $\eta=2 \%$ spontaneous emission are calculated and the difference is plotted. (b) same as (a) but with $\eta=5 \%$.

Figure 22: Comparison of classical and quantum simulations with (a) $K=$ 80, (b) 180, (c) 280 and (d) 400. Probability of finding a given atom outside $p= \pm 10 \pi$ is plotted versus number of kicks $t$. Coherent quantum evolution (light solid line); Quantum evolution with spontaneous emission rates $\eta=2 \%$ (dashed line) and 5\% (dash-dotted line) respectively. Quantum simulation with anti-Zeno effect (dotted line). Classical simulation (heavy solid line). 


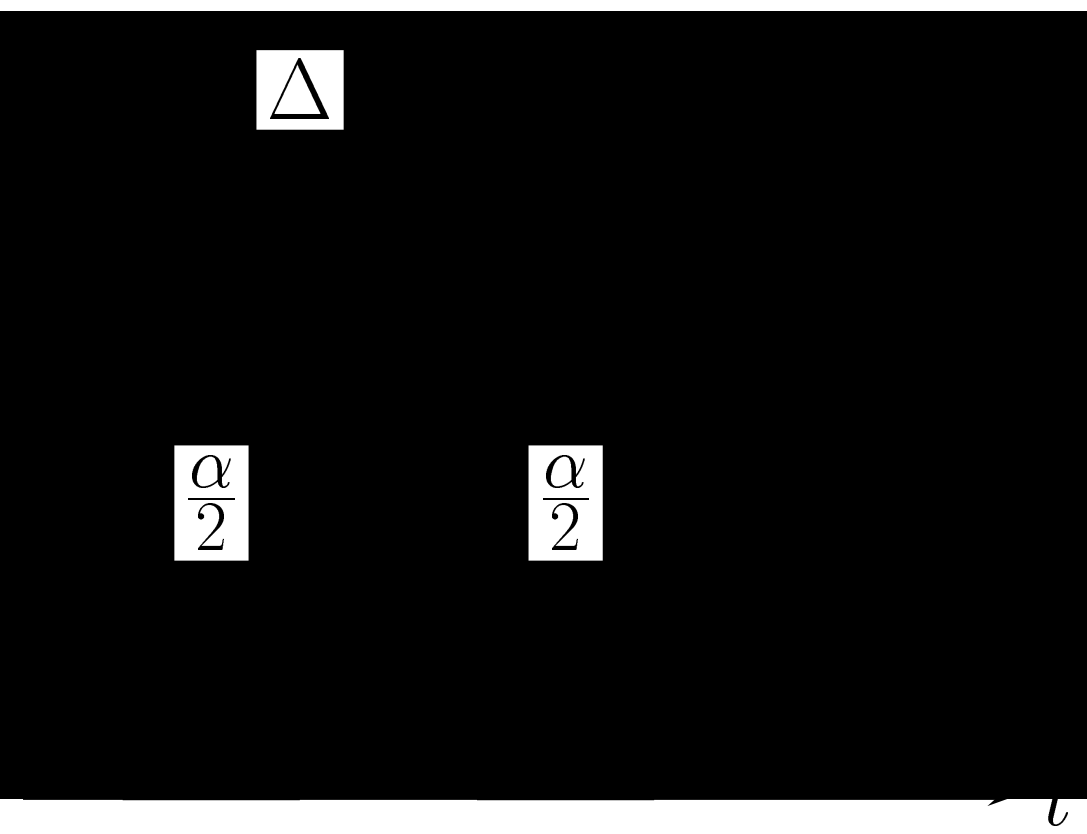




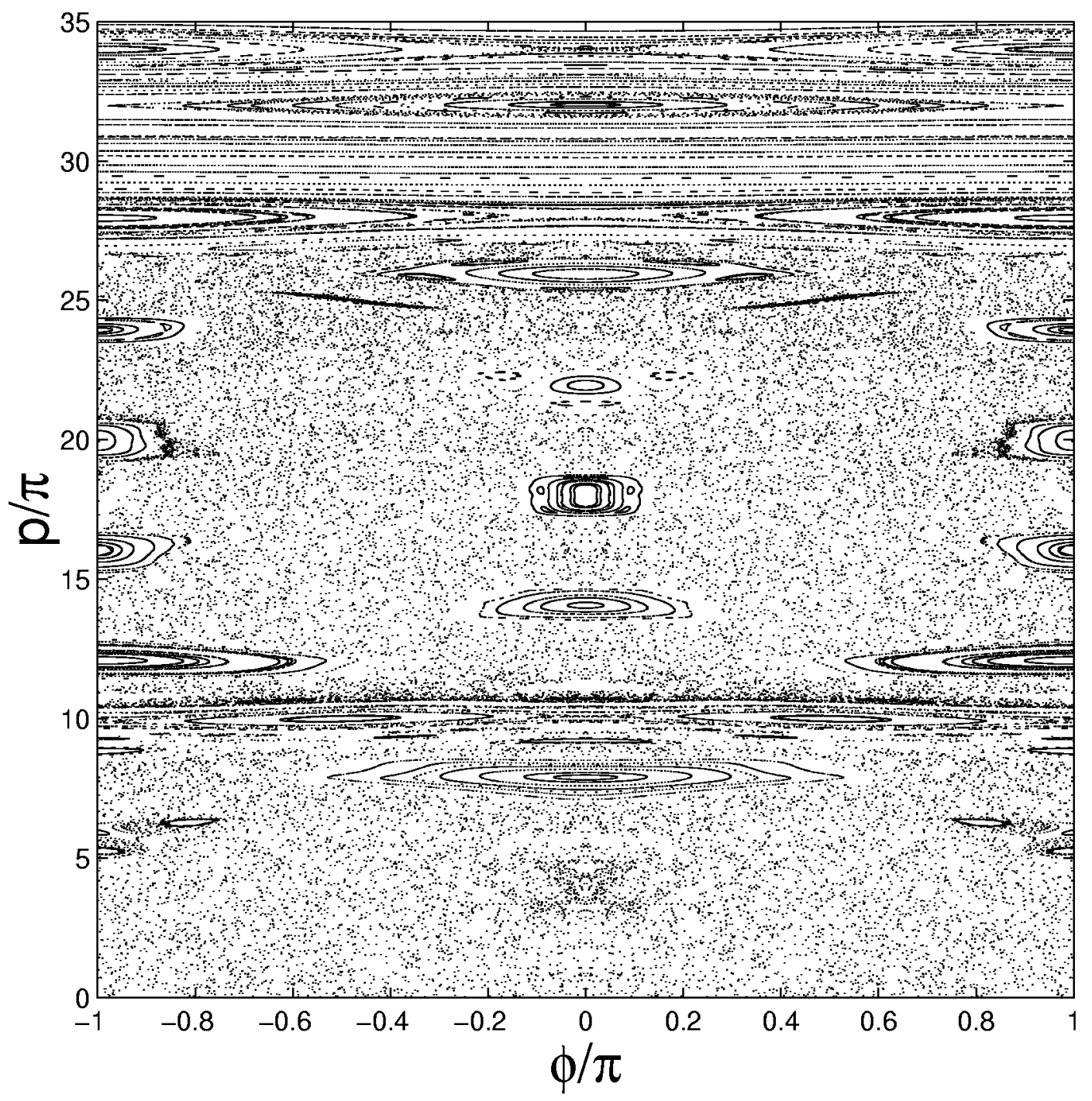




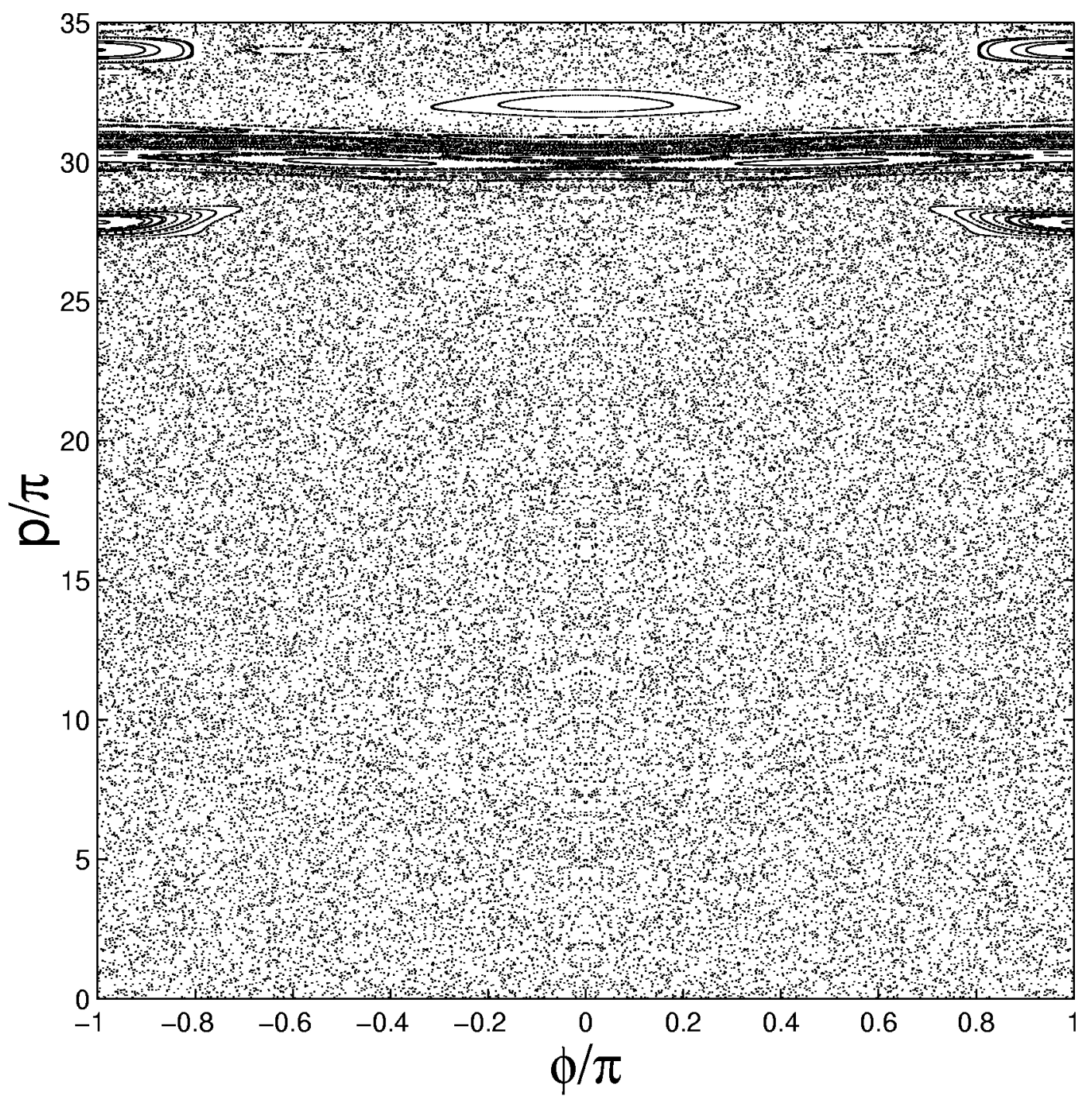


(a)

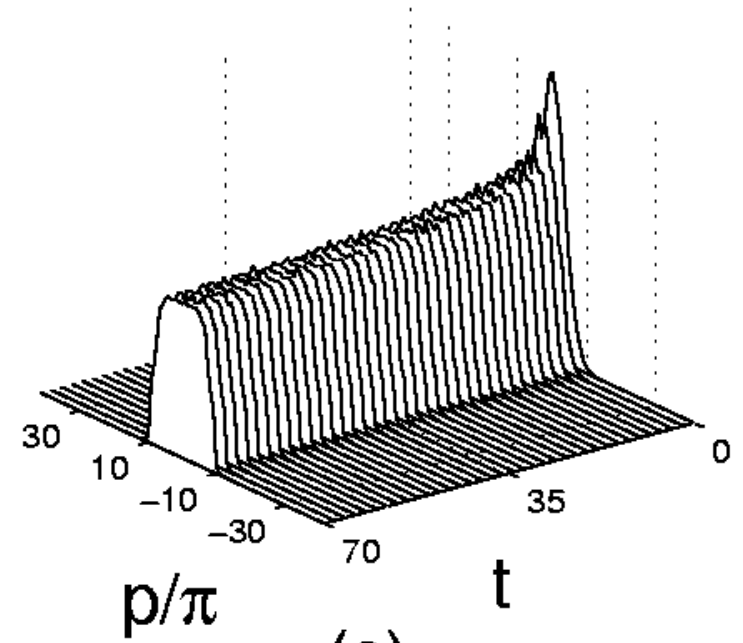

(c)

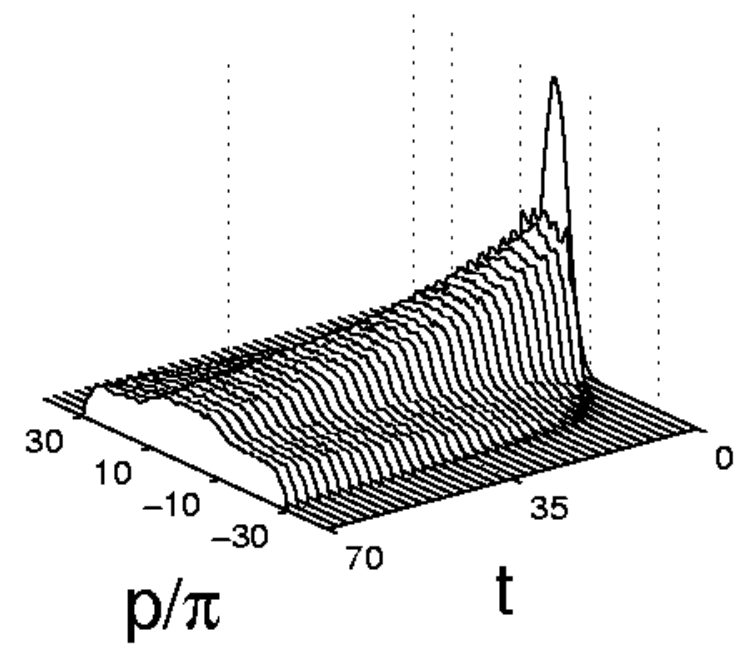

(b)

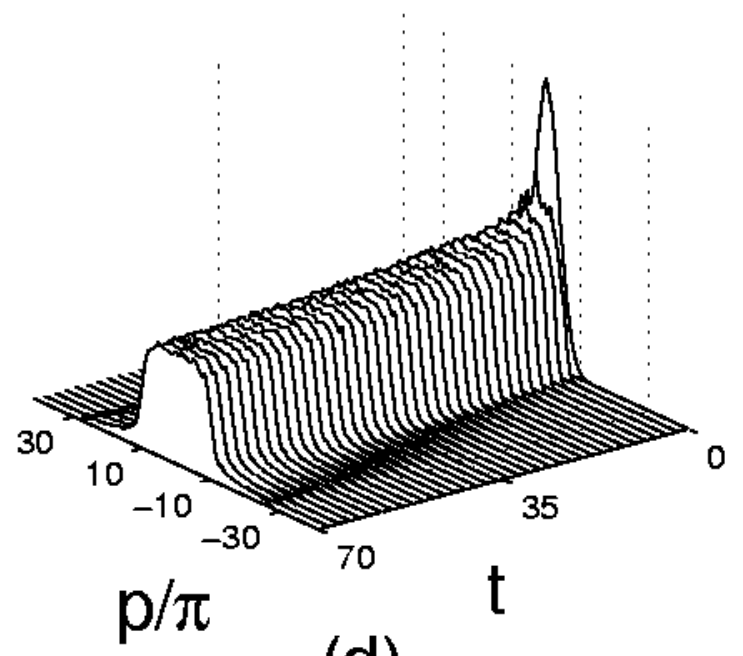

(d)

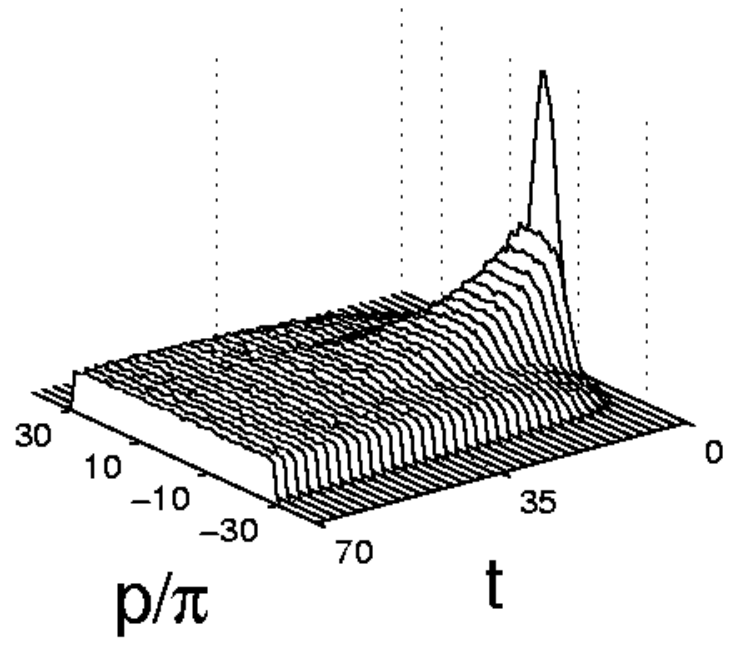


(a)

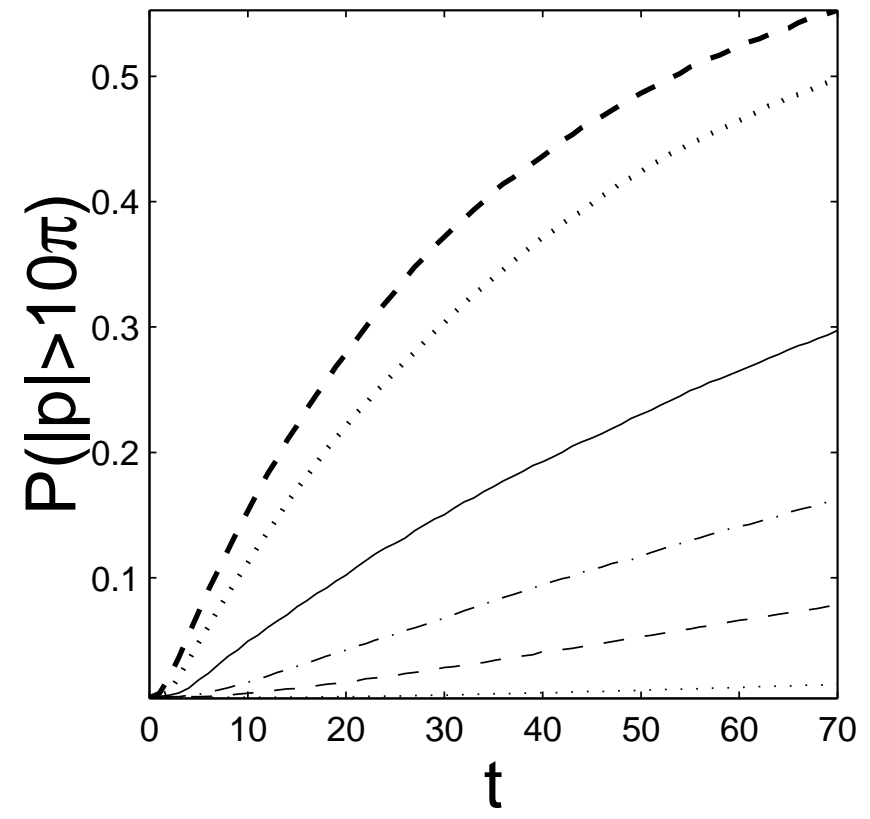

(b)

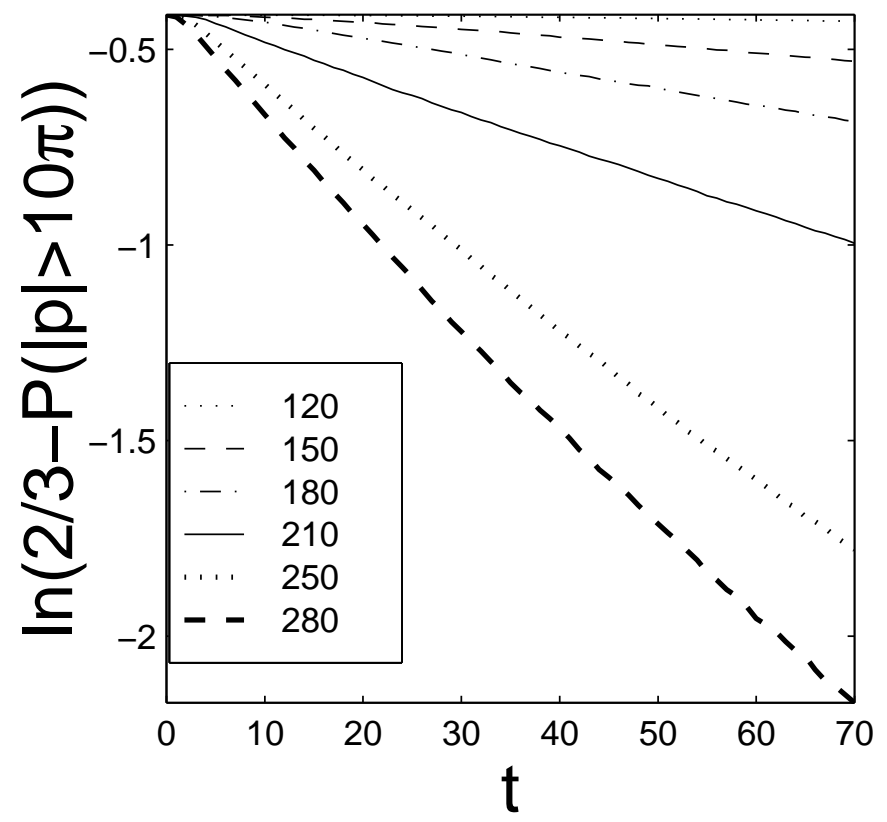




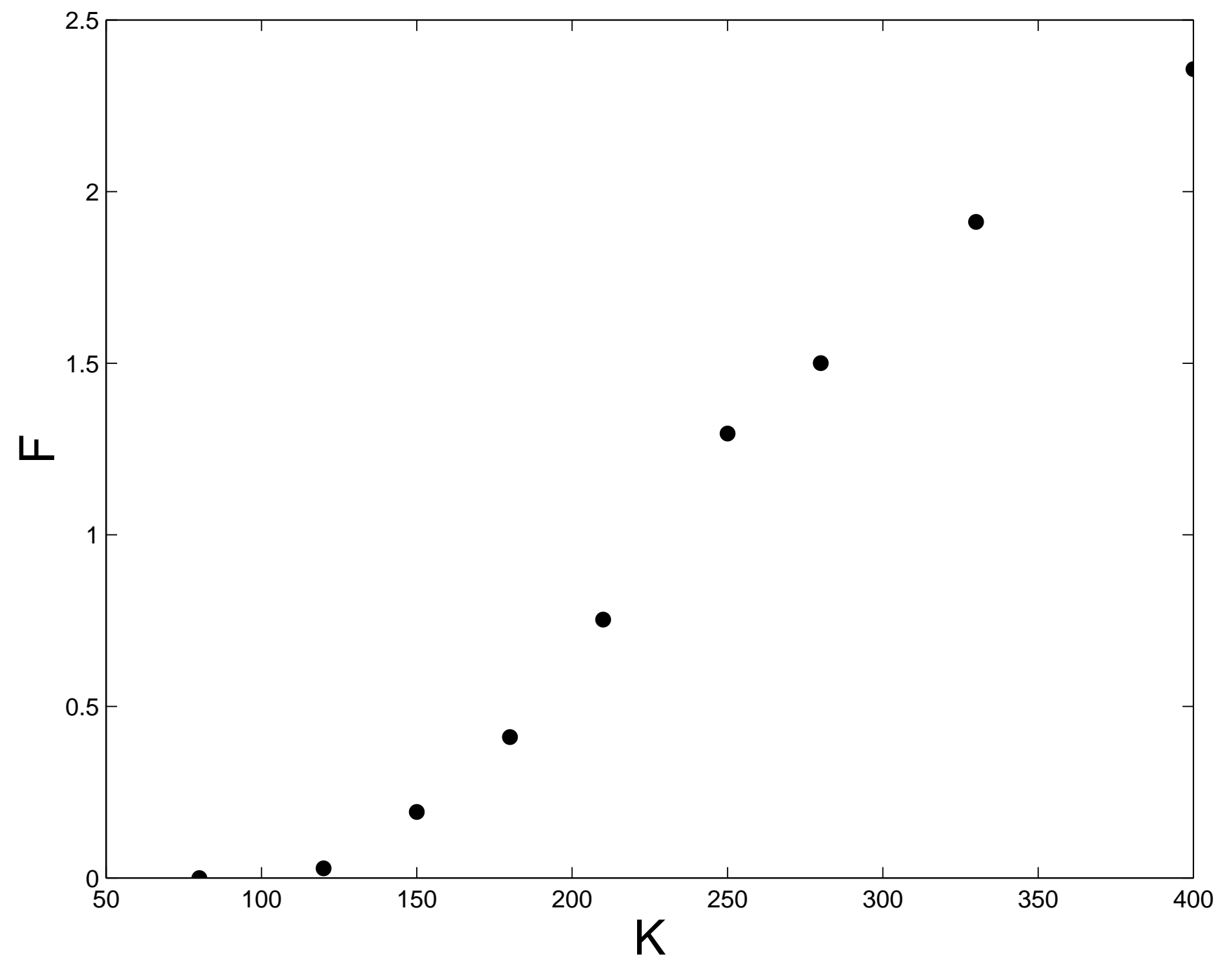


(a)

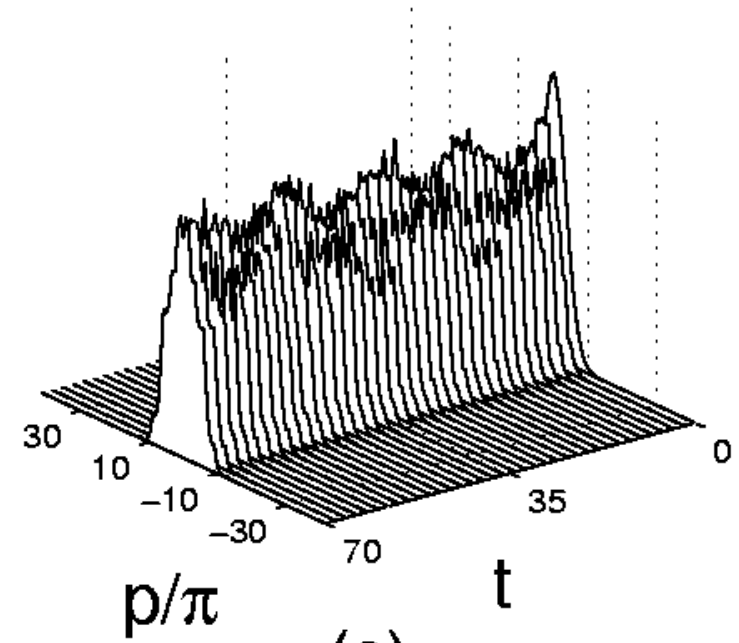

(c)

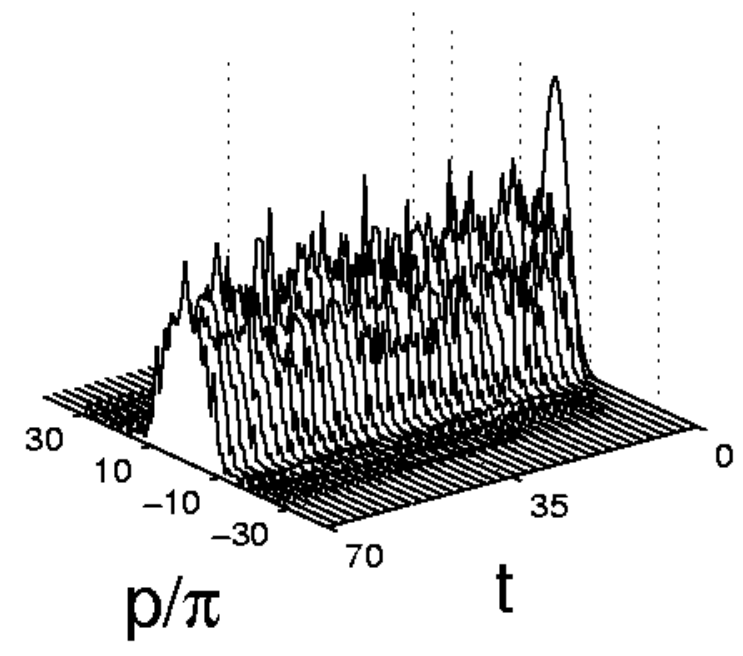

(b)

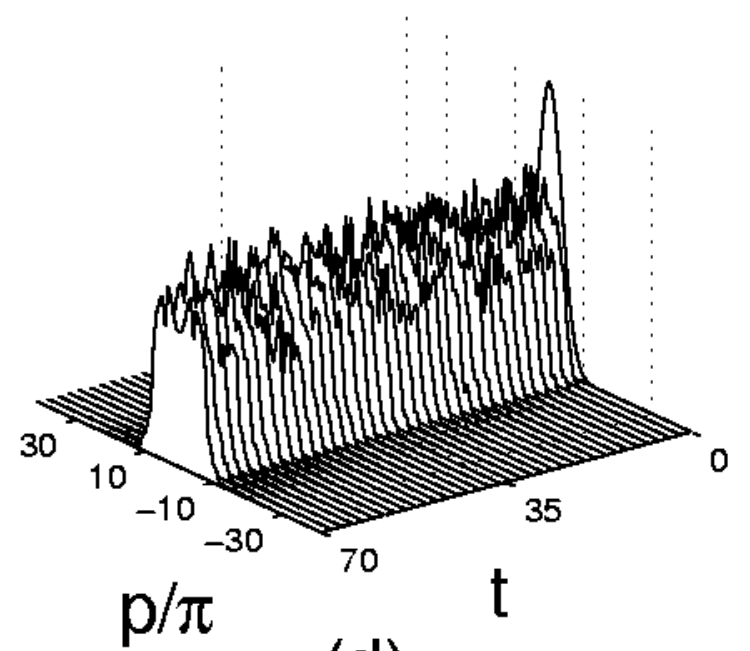

(d)

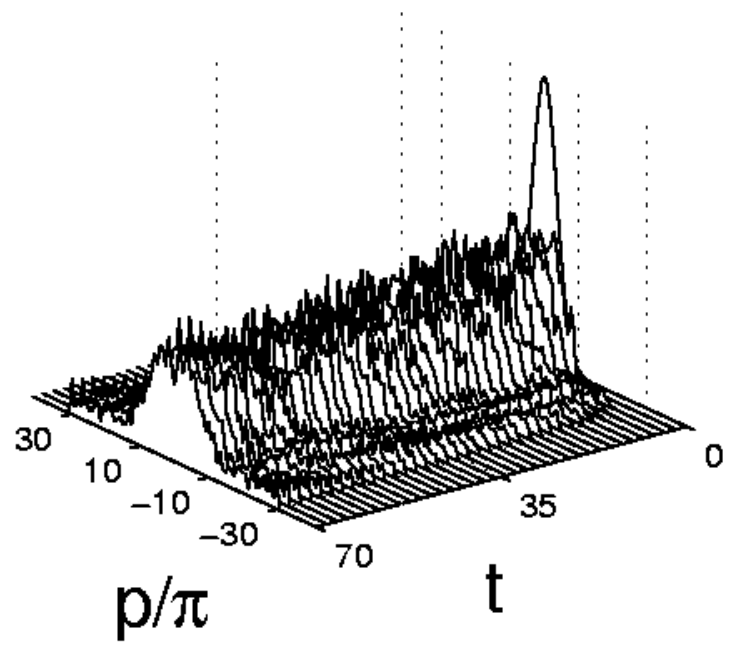


(a)

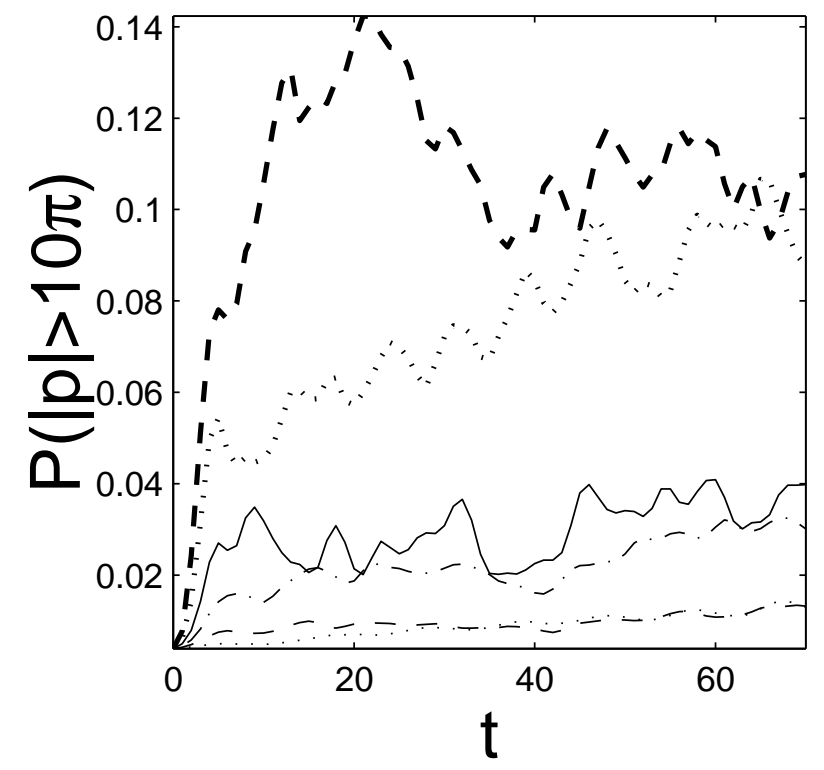

(b)

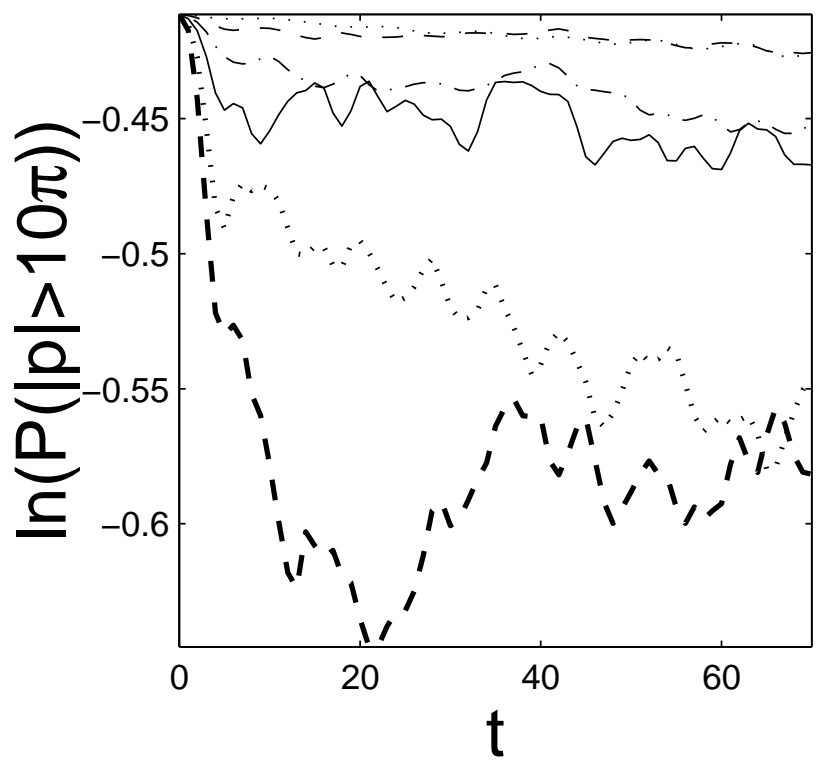


(a)

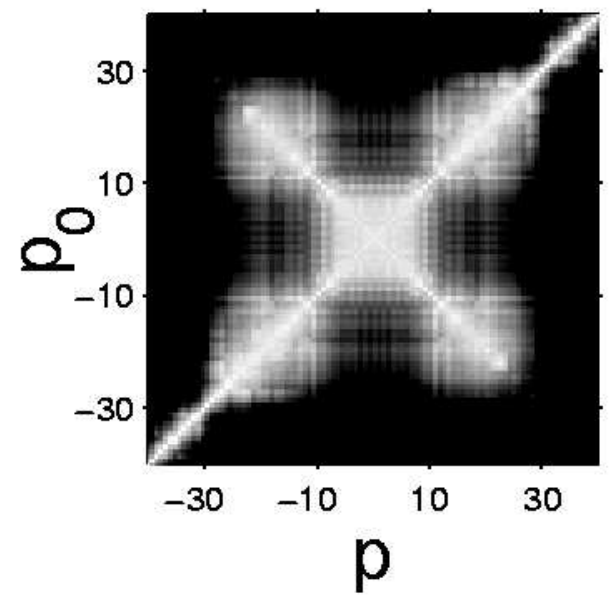

(c)

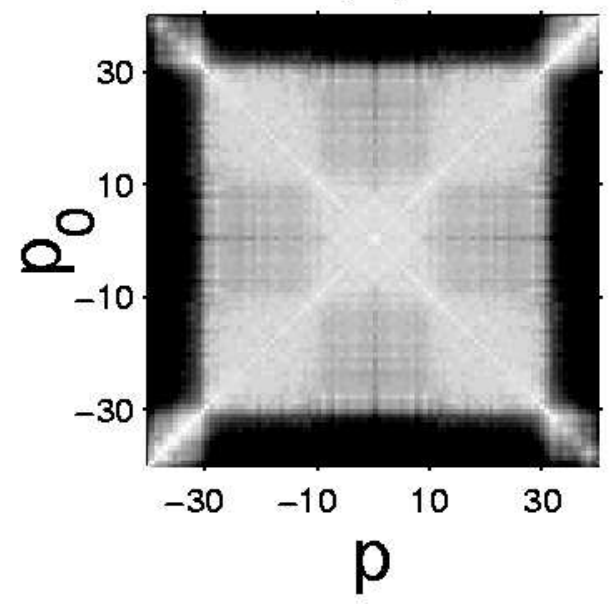

(b)

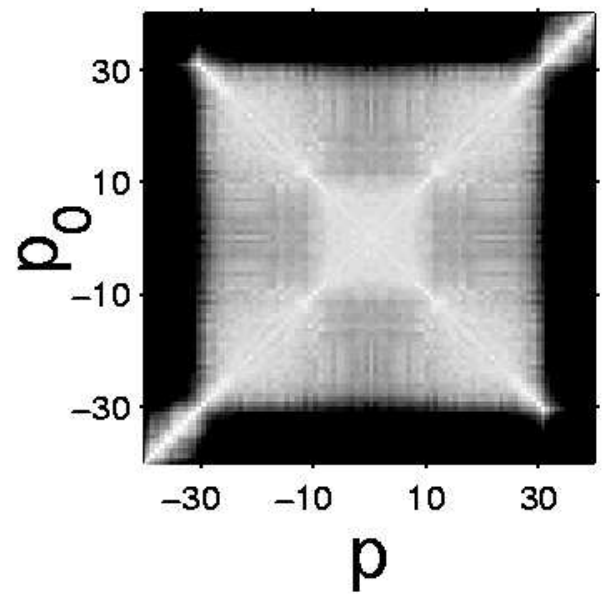

(d)

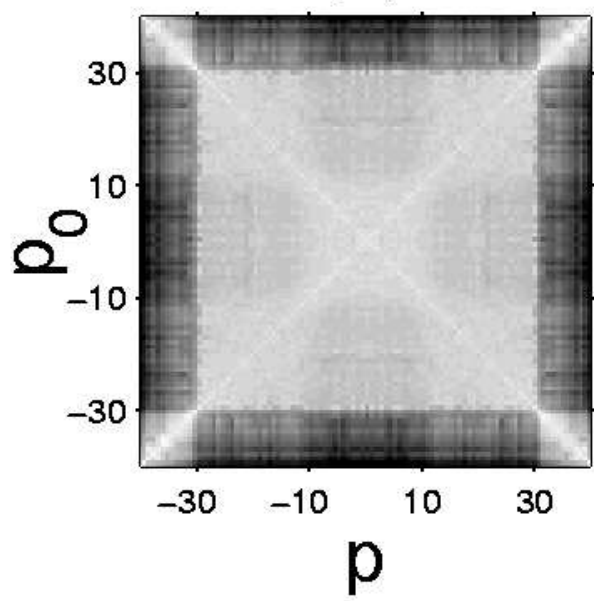

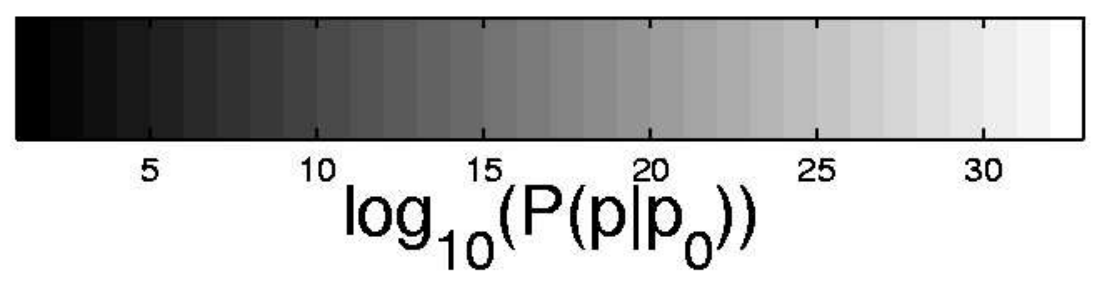


(a)

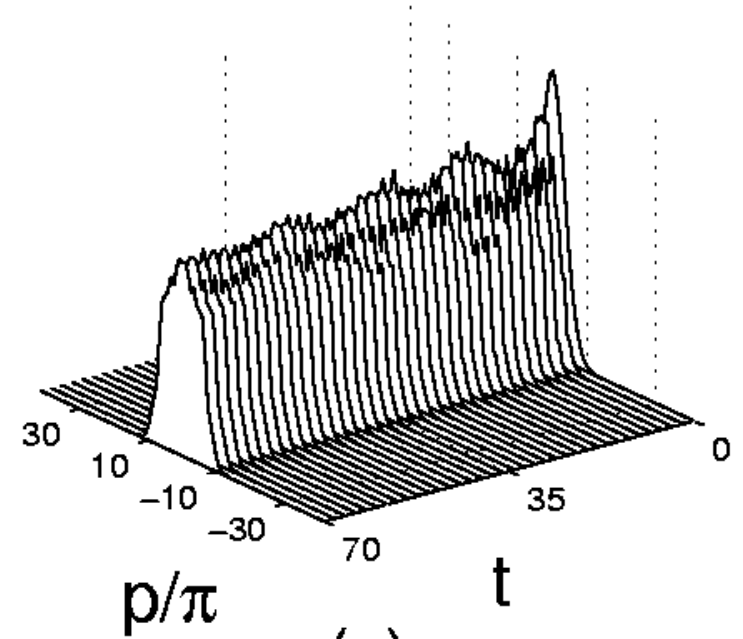

(c)

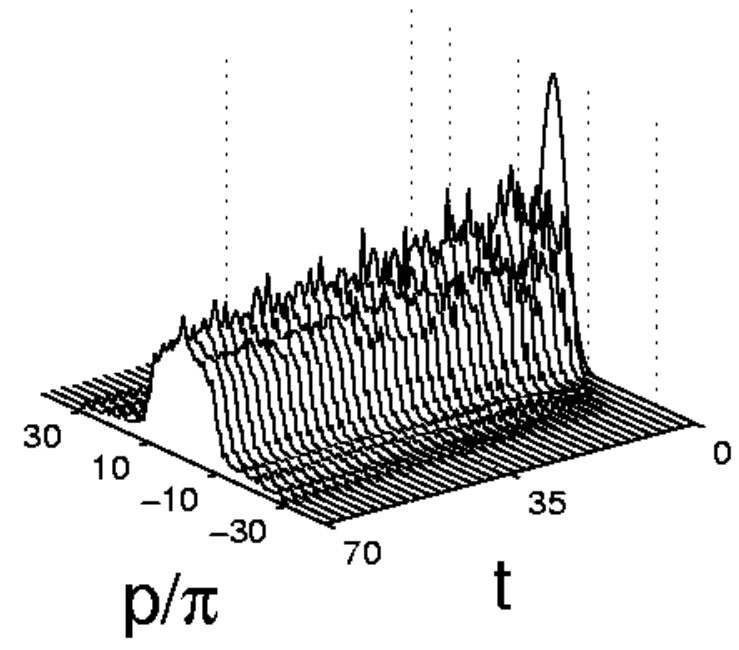

(b)

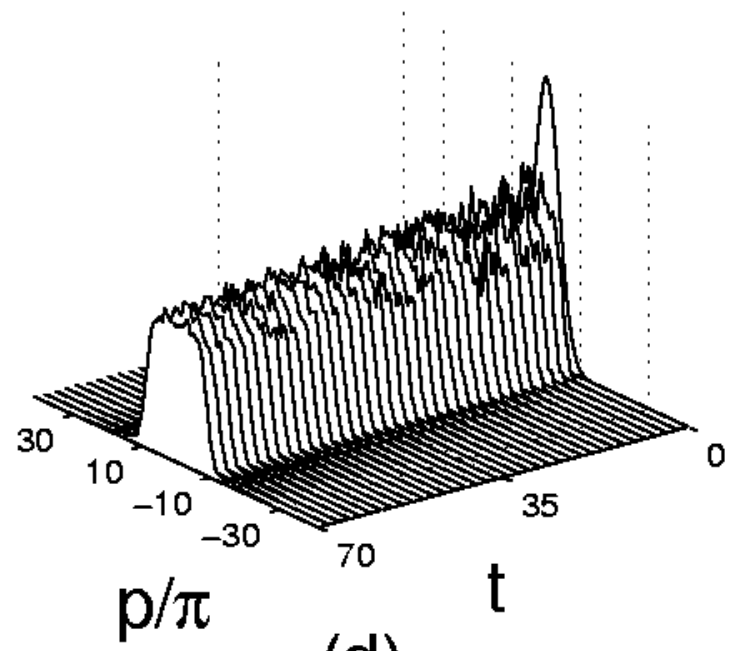

(d)

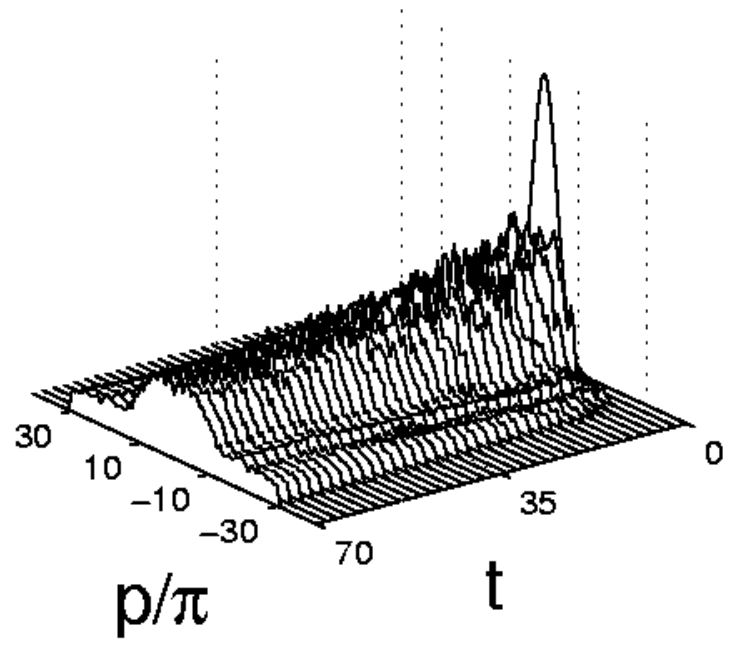


(a)

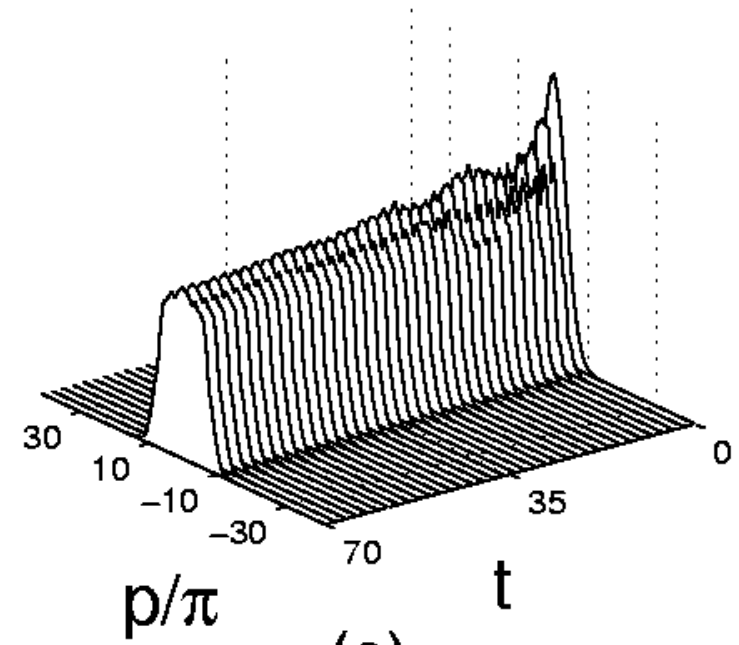

(c)

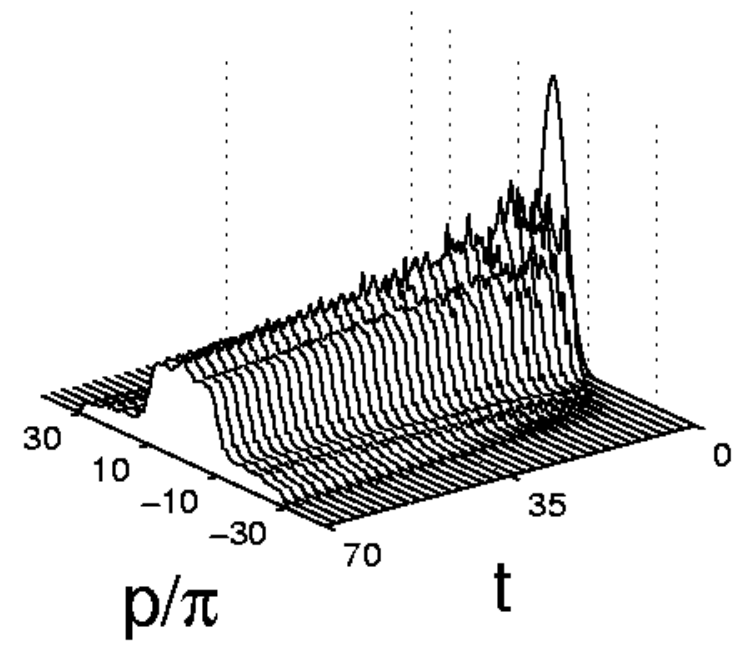

(b)

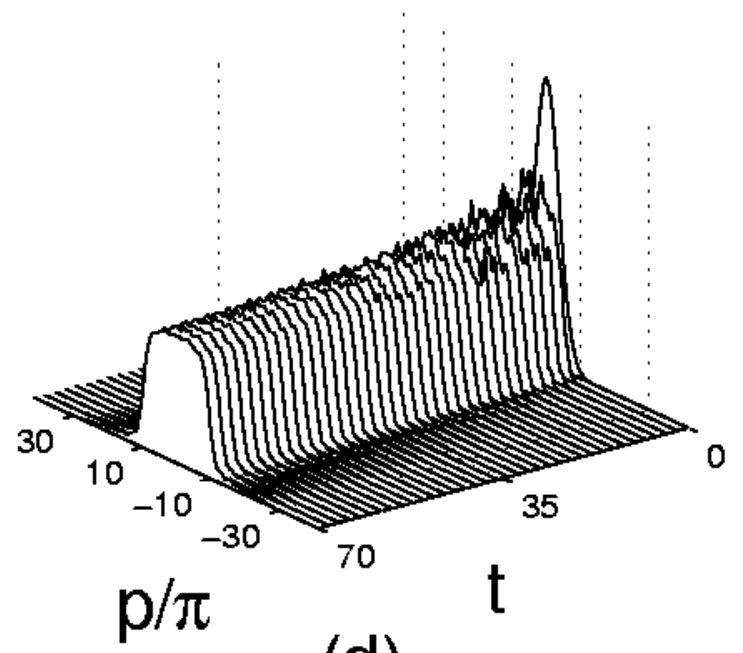

(d)

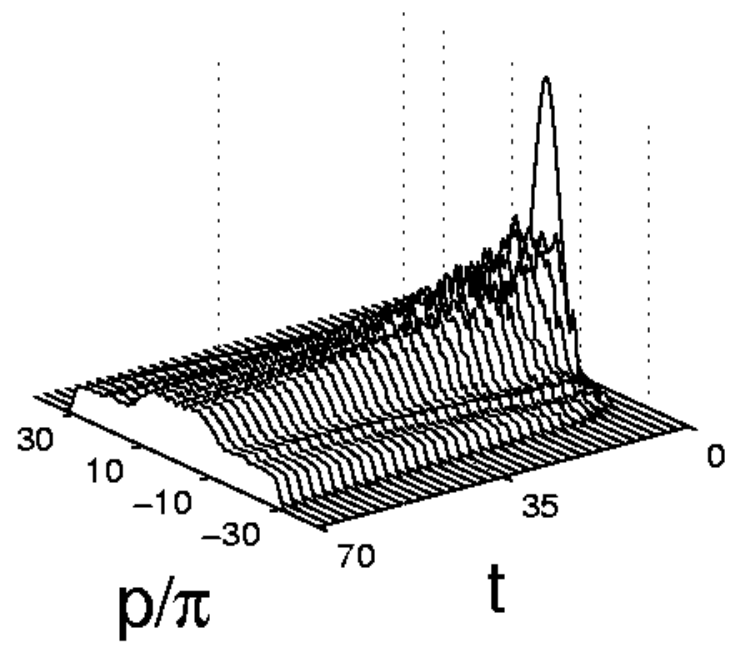




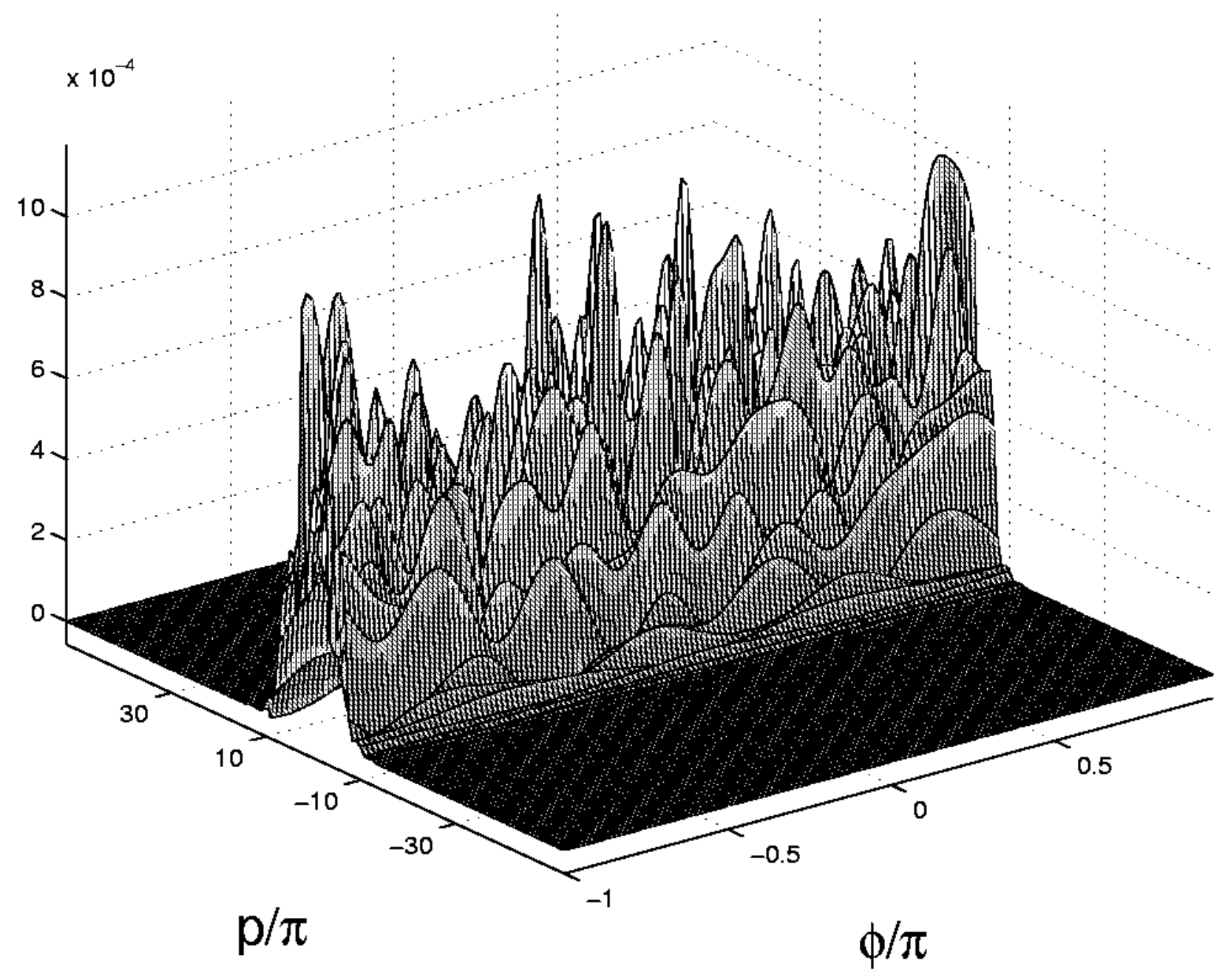




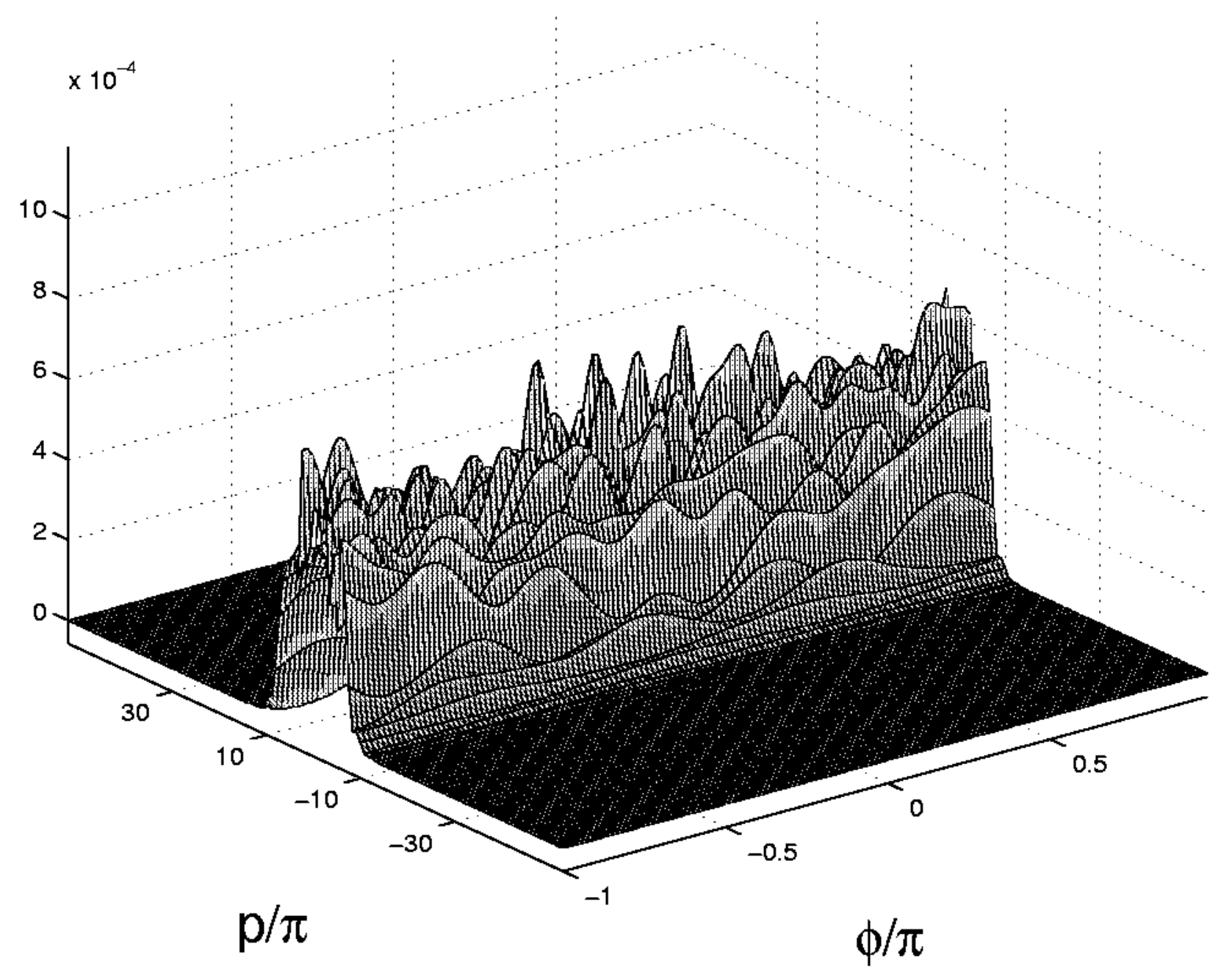




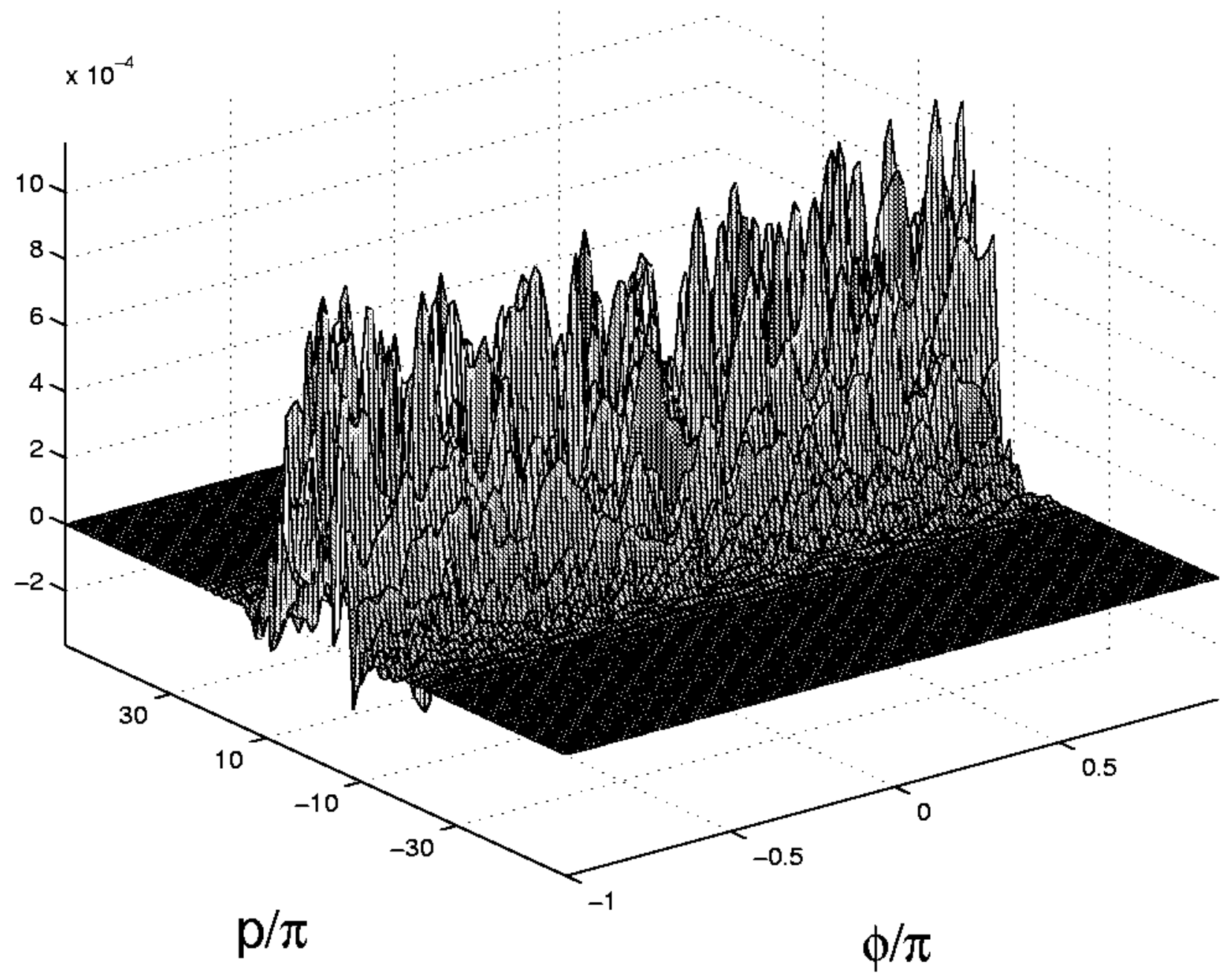




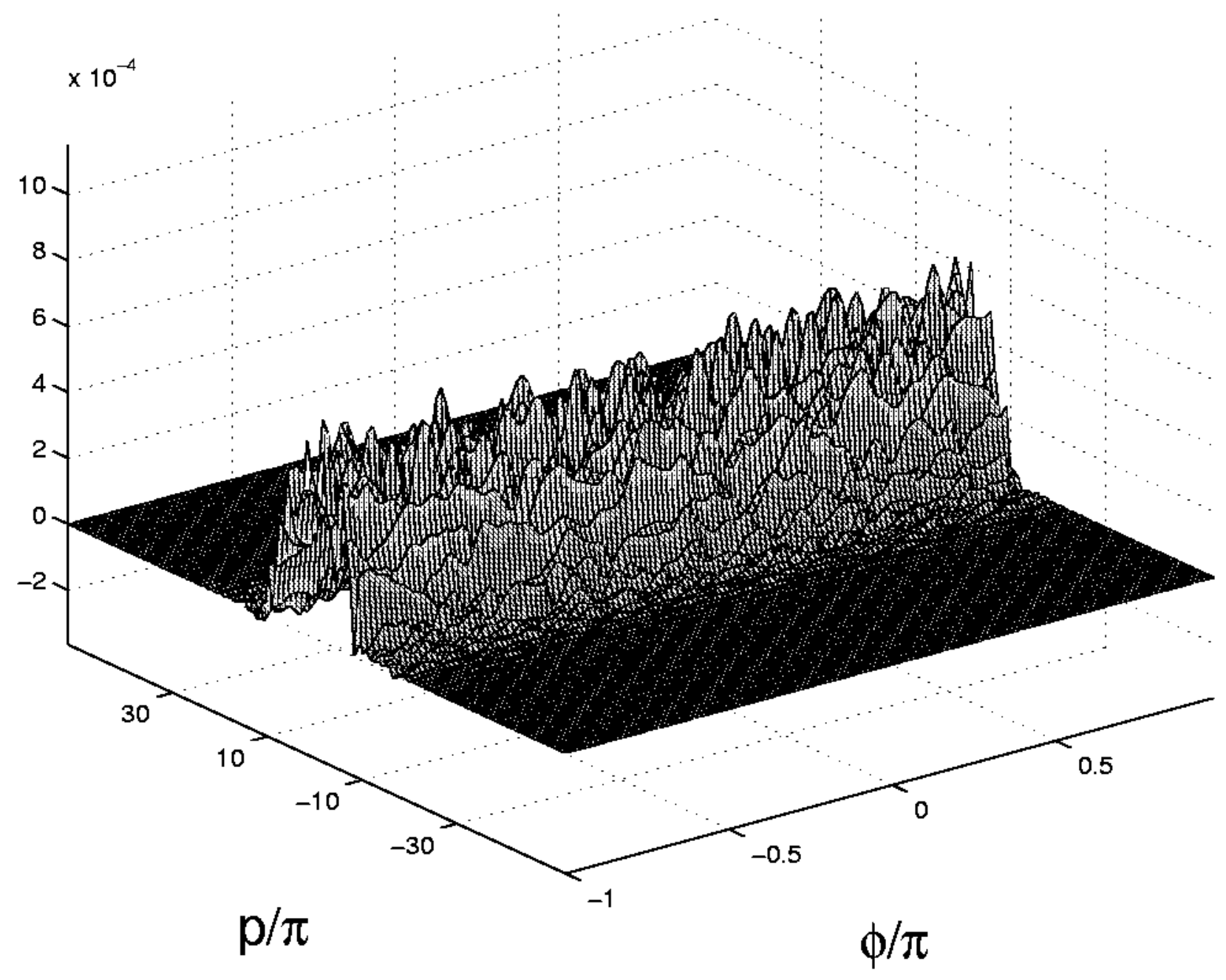




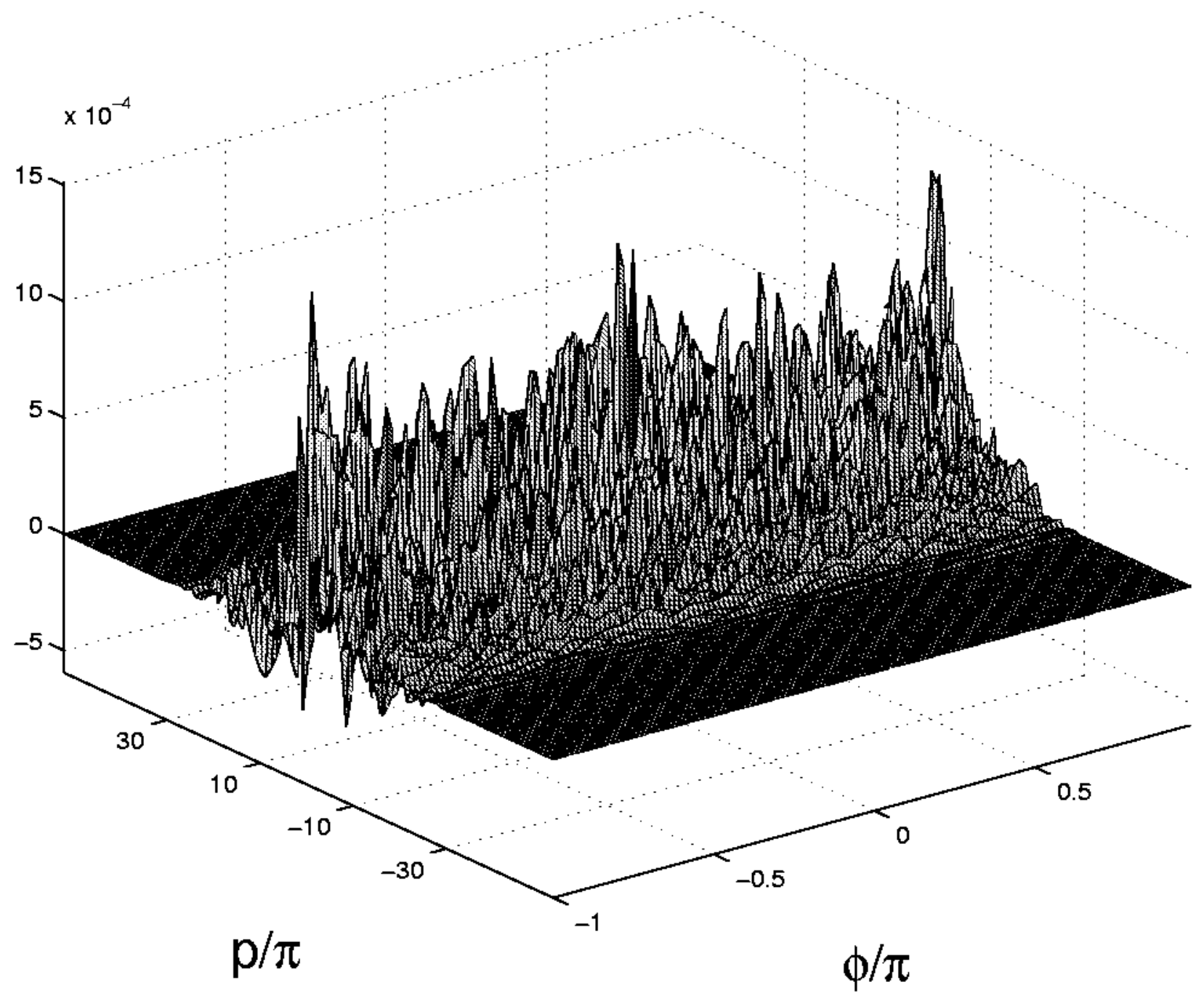




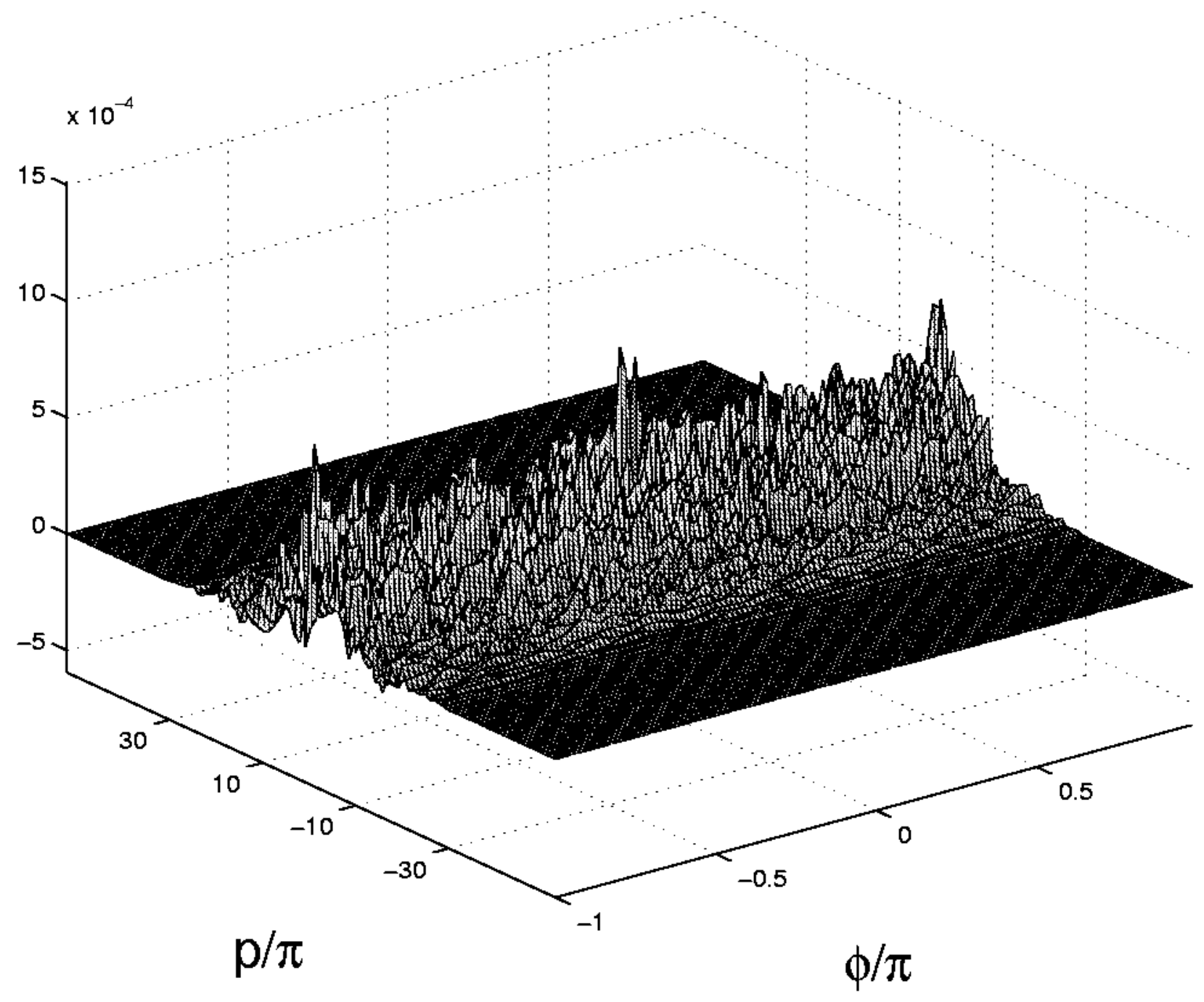




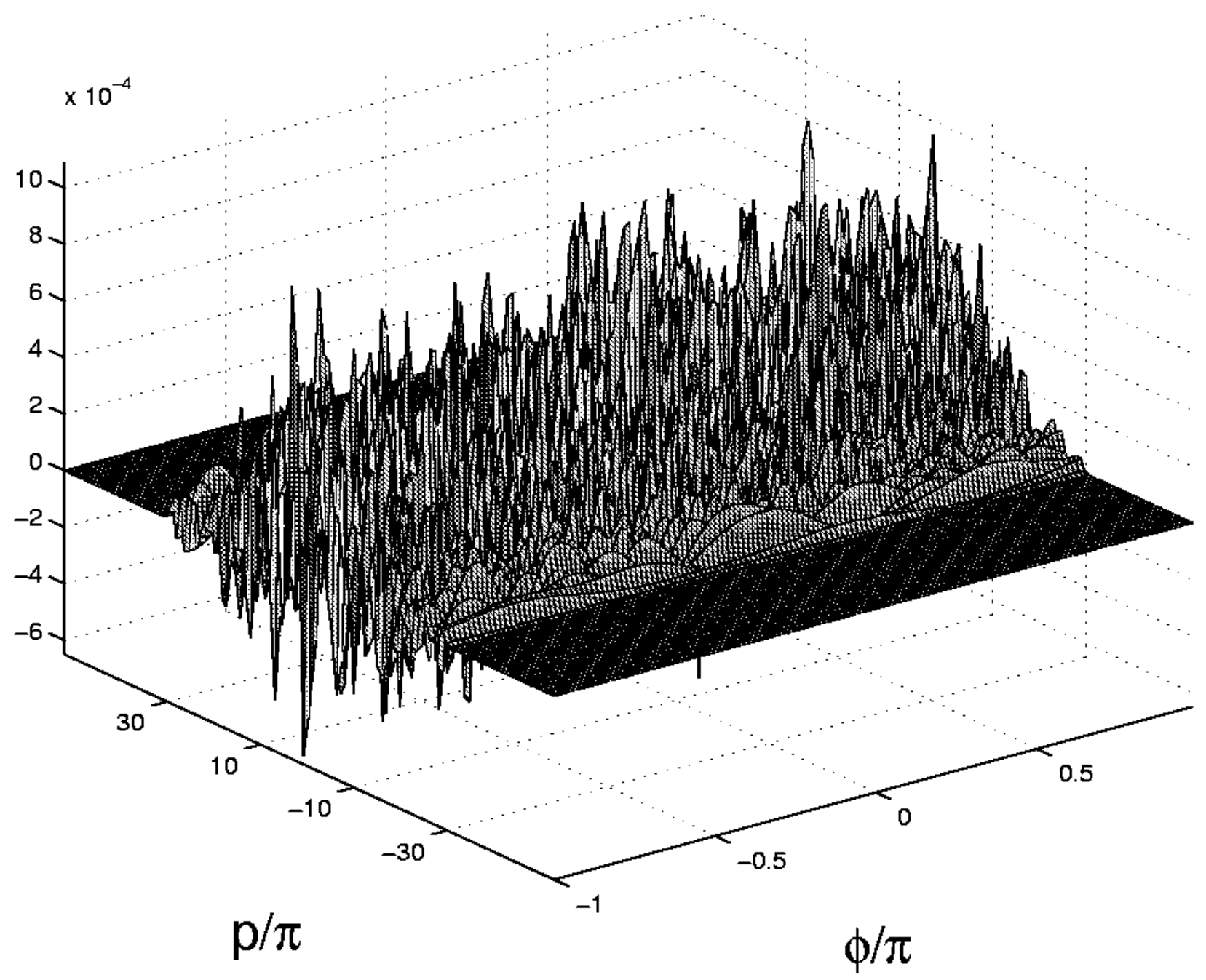




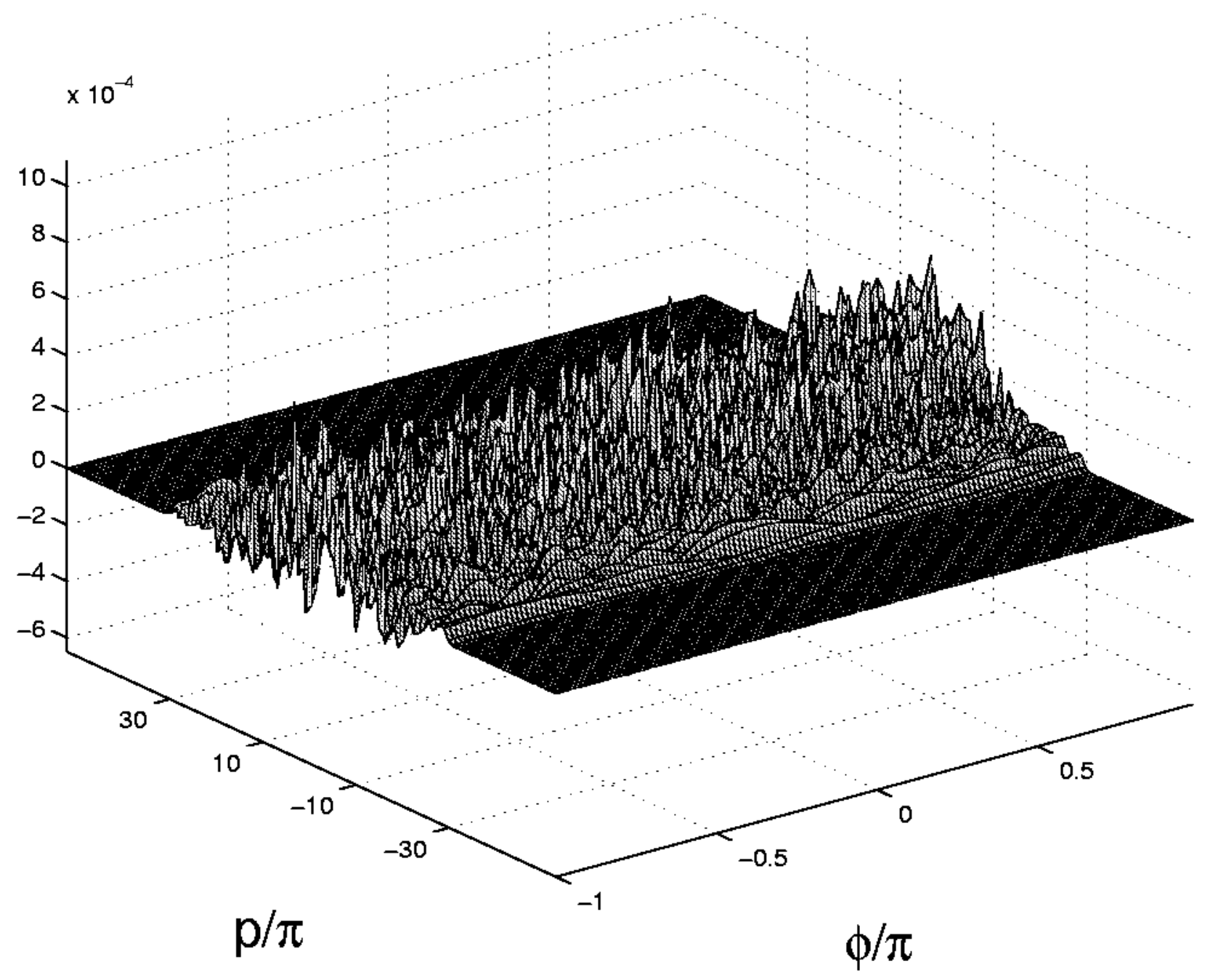




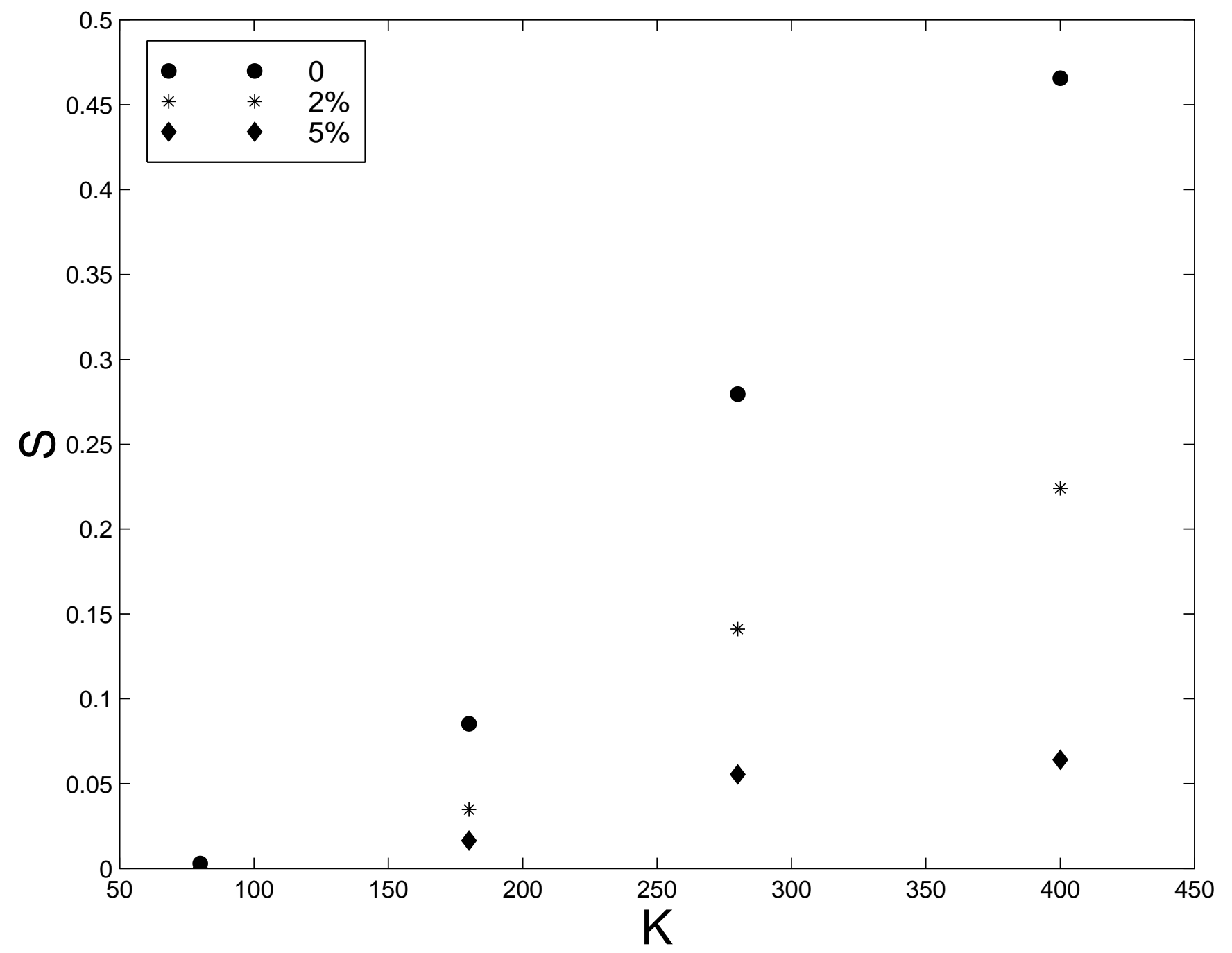


(a)

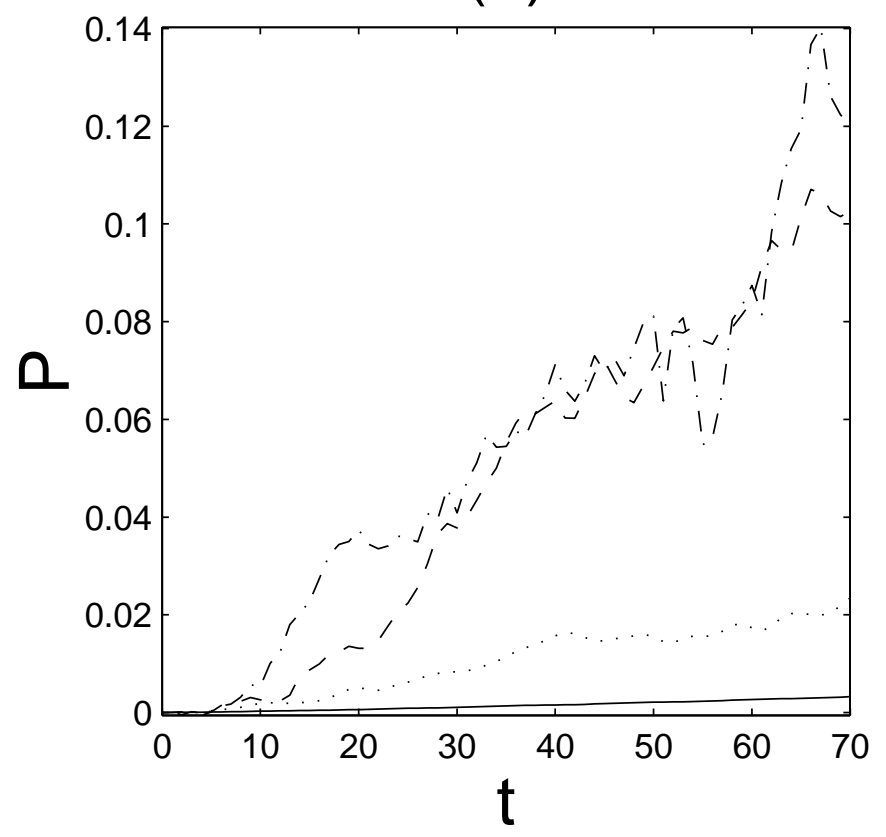

(b)

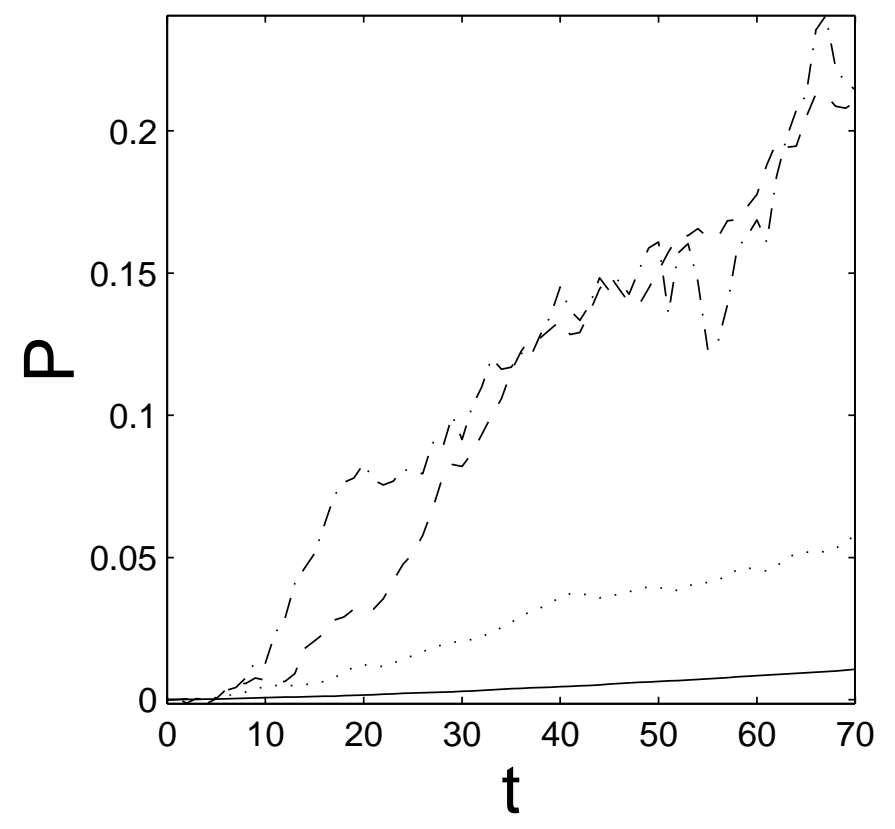


(a)

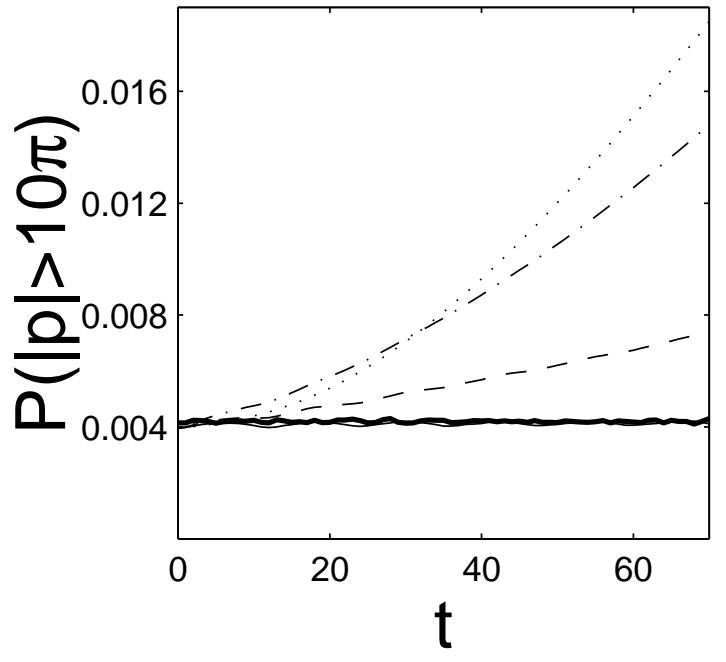

(c)

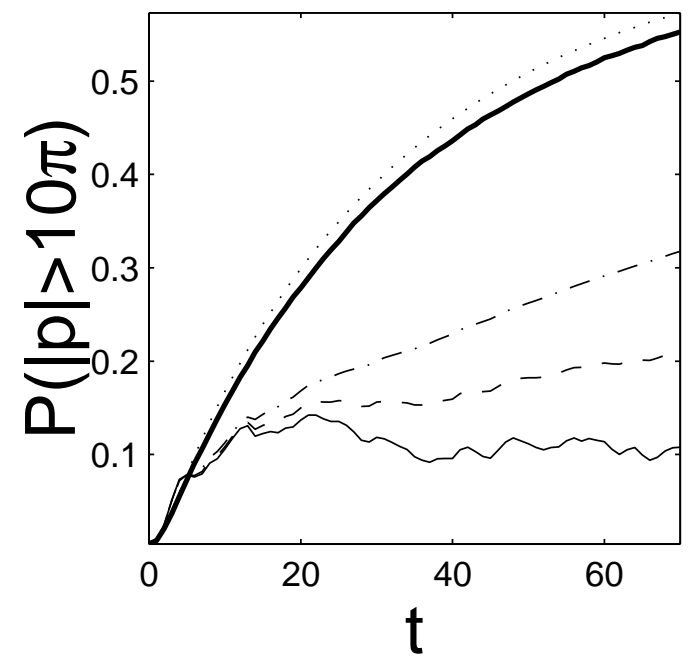

(b)

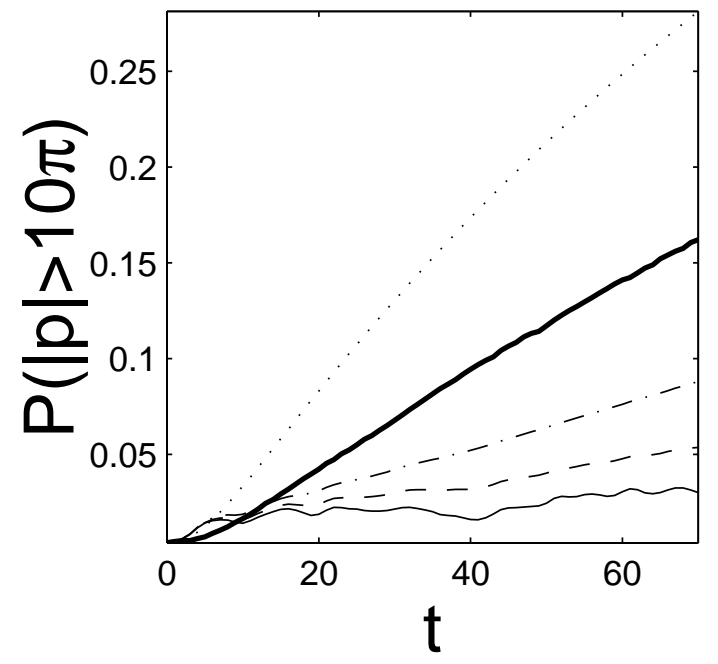

(d)

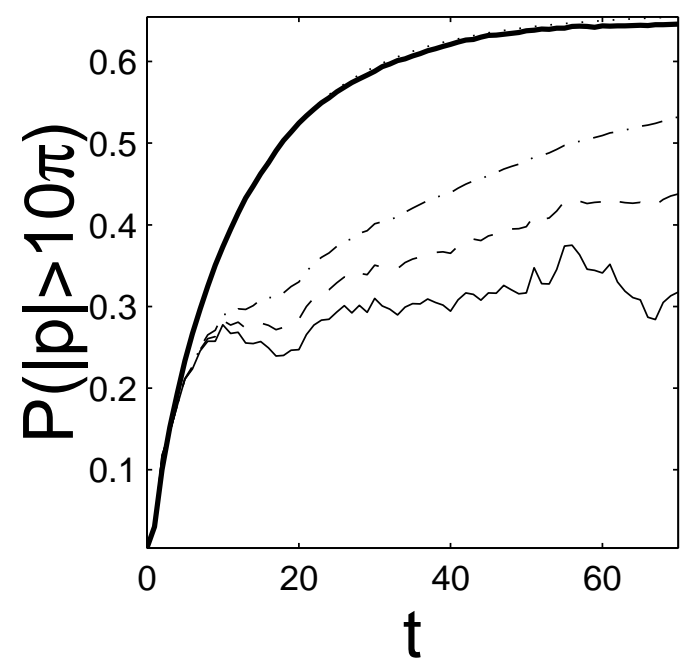

